\title{
Zur Gliederung des klastischen Holozäns im mittleren und nordwestlichen Teil der Deutschen Bucht (Nordsee) unter besonderer Berücksichtigung der Foraminiferen
}

\author{
HENNING UfFENORDE *)
}

Classification, marine sedimentation, hydrodynamic, sea floor, abrasion, tide, lithological classification, palaeoenvironment, biofacies, foraminifer fauna, cores, shoreline, continental borderland, Holocene, North Sea, German Bight. North West German Lowlands, Schleswig-Holstein, Niedersachsen.

Kurzfassung: Die stratigraphische und fazielle Gliederung der holozänen klastischen Sedimente des mittleren und nordwestlichen Teils der Deutschen Bucht wurde überprüft. Die hier vorgeschlagene Gliederung fußt auf dem von Barckhausen, Preuss \& Streif (1977) für das Küstenholozän aufgestellten lithologischen Ordnungsprinzip. Sie untergliedert die Klastische Sequenz (qhK) in folgende 3 Teilsequenzen: küstennahe Stillwasser-Ablagerungen (qhKl), küstennahe Bewegtwasser-Ablagerungen (qhK2), küstenferne Stillwasser- bis Bewegtwasser-Ablagerungen (qhK3). Die Grenzen zwischen diesen 3 Einheiten sind meist makroskopisch durch litho- und biofazielle Wechsel, teilweise sogar durch \pm deutliche Erosionsdiskordanzen, Schichtlücken und "lag-deposits" erkennbar.

Entsprechend der WALTER'schen Regel werden die Teilsequenzen paläogeographisch benachbarten Faziesräumen zugeordnet. Die Gliederung basiert auf den vorwiegend hydrodynamisch bedingten Unterschieden zwischen küstennahen Bereichen (1) mit fehlender bis geringer Wasserzirkulation und Gezeitenbewegung, (2) mit starkem Einfluß durch Wellen- und Gezeitenbewegung und (3) einem küstenfernen Bereich mit geringer, bei Stürmen starker Wasserbewegung unterhalb der Wellenbasis.

Die Basis der qhK3-Teilsequenz bildet den besten lithostratigraphischen Leithorizont im Holozän der südöstlichen Nordsee, der erosiv verschieden alten Schichten des Neogens und Quartärs auflagert. Dieser meist als Schill ausgebildete Basishorizont zeichnet sich durch hohe ResedimentGehalte aus. Die Einfrachtung allochthonen Materials spielt in der qhK2- wie in der qhK3Teilsequenz eine bedeutende, bislang vielfach unterschätzte Rolle. Unter diesem Aspekt werden die detaillierten Befunde von Kern 26 der Station 3 (KolP 1974-1976) diskutiert.

Die Untersuchung der Foraminiferen-Fauna der Teilsequenzen ergab eine Gliederung in 8 ökostratigraphische Einheiten: Ammonia batavus var.a.-Okozone. Elphidium gunteri-Ókozone, Elphidium williamsoni-Ökozone, Elphidium excavatum-Ókozone, „allochthone küstennahe Biofazies“, Ókozone der "Abroll-Foraminiferen“" (Ammonia batavus-Ökozone), Úbergangszone und Eggerelloides scaber-Ớkozone. Paraökologische (aktuopaläontologische) Verteilungsmuster liefern die Grundlage für detaillierte Interpretation des Paläo-Milieus in der Klastischen Sequenz.

\section{[On the Division of the Clastic Holocene in the Middle and Northwestern Part of the German Bight (North Sea) with Emphasis on the Foraminifera]}

A bstract: The lithostratigraphic and facial division of the Holocene clastic sediments from the middle and northwestern part of the German Bight is reviewed. The proposed division is based on the lithological classification for the coastal Holocene which has been established by Barckhausen, Preuss \& Streif (1977). In this division, the Clastic Sequence (qhK) is subdivided into the following 3 subsequences: nearshore quiet water deposits (qhK1), nearshore agitated water deposits (qhK2), and offshore quiet to agitated water deposits (qhK3). The boundaries between these 3 units are mostly recognizable macroscopically by litho- and biofacial changes, partly even by \pm distinct disconformities, stratigraphic breaks, and lag-deposits.

*) Anschrift des Autors: Dr. H. U f fen orde, Niedersächsisches Landesamt für Bodenforschung, Stilleweg 2, Postfach 510153, D-3000 Hannover 51. 
According to WALTER's rule, the subsequences are attributed to palaeogeographically neighbouring environments. The division is primarily based on different hydrodynamic conditions between nearshore regions (1) with little water circulation and tidal movements, (2) with storm wave and tidal actions, and (3) an offshore region with little water movement below wave base interrupted by storms. The base of the qhK3-subsequence constitutes the best lithostratigraphic marker horizon in the Holocene of the southeastern North Sea, which superposes Neogene and Quaternary layers of different age. This basal horizon, which is mostly developed as a shelly layer, is characterized by its high content of redeposited material. Beyond this, the influx of allochthonous material plays an important role in the qhK2- as well as in the qhK3-subsequence, a fact which has widely been neglected up to now. Under this aspect, core 26 of station 3 (KolP 1974-1976) has been reviewed.

Investigation of the foraminiferal fauna revealed a division into 8 ecostratigraphical units: Ammonia batavus var.a-ecozone, Elphidium gunteri-ecozone, Elphidium williamsoni-ecozone, Elphidium excavatum-ecozone, "allochthonous nearshore biofacies", ecozone of "abrasion-protected foraminifera" (Ammonia batavus-ecozone), transition zone, and Eggerelloides scaberecozone. Paraecological distribution patterns furnish the base for a detailled palaeoenvironmental interpretation of the Clastic Sequence.

\section{Einleitung}

Die von Barckhausen, Preuss \& Streif (1977) als Klastische Sequenz bezeichnete Sedimentabfolge im seewärtigen Teil des Küstenholozäns setzt sich im Bereich der offenen Nordsee fort und umfaßt dort meist die gesamte holozäne Schichtenfolge. Nur in wenigen Gebieten wird sie von einer Organischen Basalsequenz unterlagert.

Aus geotechnischen wie geowissenschaftlichen Gründen wird seit über 10 Jahren eine weitere Unterteilung dieser klastischen Sedimentabfolge angestrebt. So liegen lithostratigraphische Gliederungsschemata aus dem Areal SE der Doggerbank von Oele (1969, 1971 a, 1971 b), Sindowski (1970) und LABAN (1978, unveröff.) vor (s. Abb. 1). Nach Oele beginnt die Abfolge über weichselzeitlichen Sanden ("Coversands“) sowie Torfen und Süßwassertonen des Präboreals und des Boreals, die er als „Lower Peat Formation“ bezeichnete (OELE 1969: 472), mit brackischen und brackisch-marinen Tonen, Schluffen und Sanden des Präboreals und Boreals (s. Abb. 1). Diese Schichten wurden von Oele (1969) zunächst als „Elbow-deposits“, später als „Elbow/Calais-deposits“ (Oele 1971) bezeichnet, da sie in der küstennahen Zone ins Atlantikum gestellt werden (s. auch LABAN 1978, Jelgersma et al. 1979). Die - offenbar mit Hiatus - darüber lagernden gelblichbraunen Sande nannte Oele „Young Seasand“. Sie werden nach Jelgersma et al. (1979) mit den marinen grauen Sanden vor Seeland zu den „Dunkerque deposits“ zusammengefaßt. Der in die grauen Sande eingeschaltete Ton ist nach Pollen dem Subatlantikum zuzuordnen; Jelgersma et al. geben ein Alter zwischen 2000 und $500 \mathrm{~J}$. v. h. an. Der Junge Seesand wurde erstmals von BeHre \& Menke (1969) ins Subatlantikum, sein oberster Teil mit Getreide-Pollen als mittelalterlich oder jünger eingestuft. Eine neuere Altersangabe aus dem Jungen Seesand des niederländischen Nordseesektors liegt von JELGERSMA et al. (1979) vor. Danach wurden Ton-Lamellen, die in die Jungen Seesande im nördlichen Teil des Sektors eingeschaltet sind, als spätmittelalterlich datiert.

Die Gliederung des Holozäns im deutschen Nordseesektor von SindowskI (1970) schloß sich im wesentlichen der niederländischen Gliederung an. Aus Mangel an Altersdatierungen unterschied er die Schichten des fraglichen Calais von denen des Dünkirchen palökologisch aufgrund ihrer Molluskenfauna. Den rezenten Verhältnissen entsprechend unterschied er zwischen der "Macoma baltica-Gesellschaft“ der küstennahen Sandgebiete bis $15 \mathrm{~m}$ Wassertiefe und der „Venus gallina-Gesellschaft" der küstenfernen Sandgebiete in 15-20 m Wassertiefe und der Schlicksand- und Schlickgebiete in 25-50 m Wassertiefe, wobei er die Schichten mit "Macoma baltica-Gesellschaft" dem fraglichen Calais und die Schichten mit „Venus gallina-Gesellschaft“ dem Dünkirchen zuordnete. Die von Sindows- 
KI untersuchten Bohrungen enthielten keine spätglazialen und holozänen Torfe oder Mudden. Die oberen marinen Schichten lagern unmittelbar auf weichselzeitlichen „limnischfluviatilen Schichten “. Möglicherweise wurden geringmächtige Brackwasser-Sedimente mit zu den marinen Schichten gerechnet.

\section{Gliederung des Holozäns im offshore-Bereich}

\subsection{Verknüpfung mit dem Küstenholozän}

Die hier vorgeschlagene Gliederung des Holozäns im mittleren und nordwestlichen Teil der Deutschen Bucht (s. Abb. 2) kann z. Zt. noch nicht unmittelbar an das von Barckhausen, Preuss \& Streif (1977), Streif (1978/79) dargestellte lithologische Ordnungsprinzip anschließen, da die Untersuchungen im nearshore-Bereich bislang eine gesicherte Korrelation im Detail nicht erlauben.

Barckhausen et al. unterschieden im Bereich des Küstenholozäns 3 Komplexe, die als Mineralischer Komplex (qhMK, Symbol in Ergänzung zu BARcKHAusen, Look, VinkeN \& Voss 1975), Verzahnungskomplex (qhVK) und Torfkomplex (qhTK) bezeichnet wurden. Die Fortsetzung des Mineralischen Komplexes besteht - im Küsten- wie im offshoreBereich - überwiegend aus klastischen Ablagerungen ( $\mathrm{qhK}=$ Klastische Sequenz). Nur örtlich werden diese auch von Torfen, Mudden oder Ah-Horizonten eines fossilen Bodens unterlagert $(\mathrm{qhOB}=$ Organische Basalsequenz).

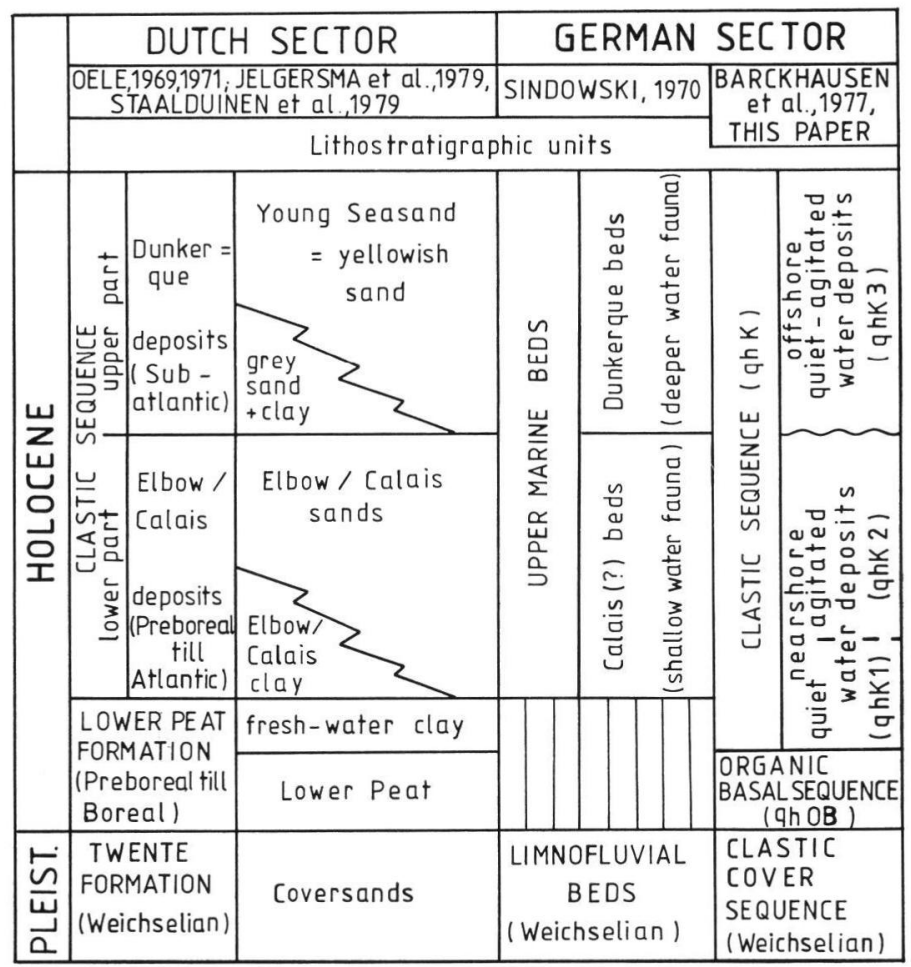

Abb. 1: Vergleich der lithostratigraphischen Gliederungsschemata des Holozäns im niederländischen und deutschen Nordseesektor.

Text-Fig. 1: Comparsion of the lithostratigraphic schemes of the Holocene in the Dutch and German North Sea sectors. 


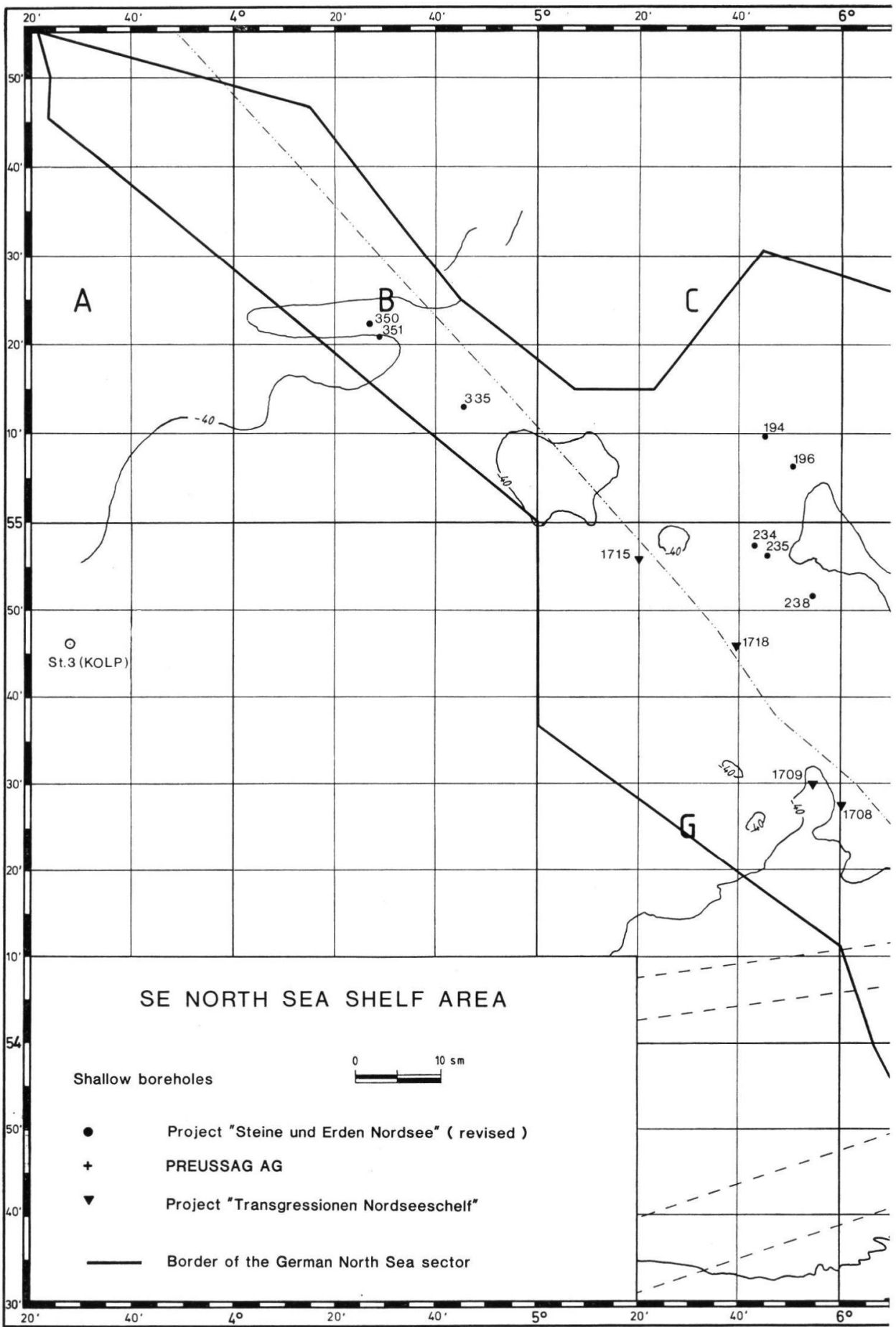

Abb. 2: Lage der im Text erwähnten Bohrungen im deutschen Nordseeschelf-Anteil.

Text-Fig. 2: Position of boreholes, mentioned in the text, in the German part of the North Sea shelf area. 


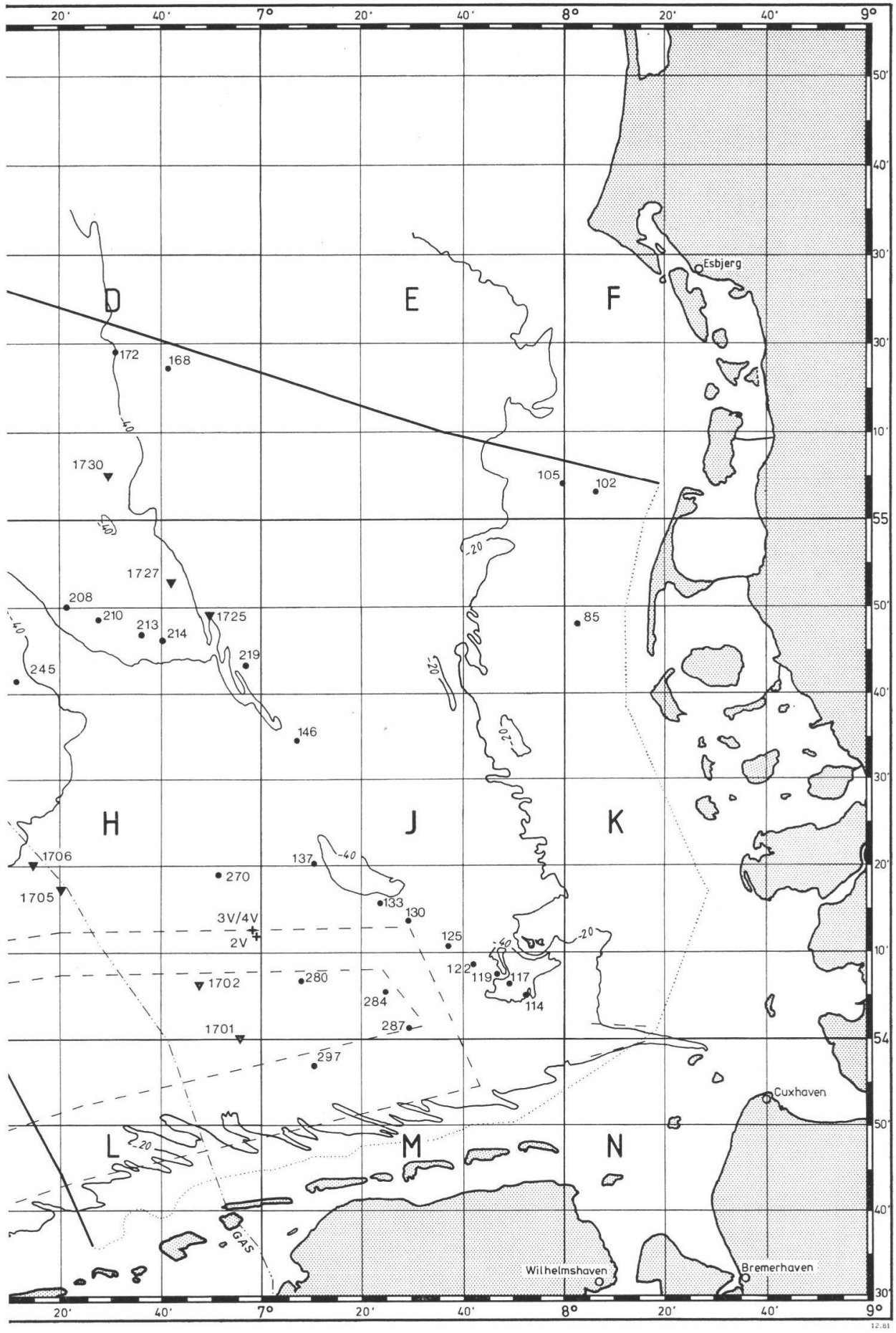


Die z. Zt. laufenden Untersuchungen im deutschen Nordseesektor (STREIF \& UfFeNORDE 1980, unveröff.) machen eine Unterteilung der im offshore-Bereich dominierenden Klastischen Sequenz notwendig, um (1) das holozäne Transgressionsgeschehen, (2) unterschiedliche Faziesentwicklungen und (3) die Mächtigkeitsverteilung von Teilsequenzen und Fazieseinheiten verfolgen und darstellen zu können. Auf den nicht nur geowissenschaftlichen, sondern auch geotechnischen Nutzen einer Unterteilung in Fazieseinheiten hat insbesondere STREIF $(1978,1979)$ hingewiesen.

Die Teilsequenzen stehen dem Range nach zwischen Sequenz und Fazieseinheit (i. S. von BARCKHAUSEN et al. 1977), da sie einerseits die Klastische Sequenz untergliedern, andererseits aber mehrere Fazieseinheiten enthalten können.

\subsection{Gliederung der Klastischen Sequenz (qhK)}

Im mittleren und nordwestlichen Teil der Deutschen Bucht lassen sich in der Klastischen Sequenz drei Teilsequenzen auseinanderhalten (s. Abb. 1) : (1) küstennahe Stillwasser-Ablagerungen (qhK1), (2) küstennahe Bewegtwasser-Ablagerungen (qhK2) und (3) kästenferne Stillwasser- bis Bewegtwasser-Ablagerungen (qhK3). Die Grenzen zwischen den drei Faziesbereichen sind meist durch makroskopisch erkennbare litho- und biofazielle Wechsel, teilweise sogar durch \pm deutliche Erosionsdiskordanzen, Schichtlücken und „lagdeposits" charakterisiert. Abb. 2 zeigt die Lage der in der vorliegenden Arbeit angeführten Bohrungen im deutschen Nordsee-Schelfanteil.

\subsubsection{Küstennahe Stillwasser-Ablagerungen (qhKl)}

In der qhKl-Teilsequenz werden alle küstennahen Süß-, Brack- und MeerwasserAblagerungen zusammengefaßt, die bei fehlender oder schwacher Wasserbewegung entstanden. Die fazielle Ausbildung entspricht der letal-pantostraten bis vital-pantostraten Biofazies (SCHÄFER 1963). Die Sedimente bestehen vorwiegend aus dunklen Tonen und Schluffen mit einzelnen humosen Lagen, Schilfdurchwurzelungshorizonten (Schilfdurchwurzelungsgrad $<$ hpr 4, d. h. < stark durchwurzelt), Torfdetritus-Lagen und (par-)autochthonen Fossil-Lagen. Gelegentlich finden sich Lagen von Molluskenschill und gröberklastischen Sedimenten, die möglicherweise von Sturm-Ereignissen herrühren. Meist sind die im unteren Teil der Folge nachgewiesenen Süßwassertone kalkfrei. Die kalkigen Partien zeigen generell eine lagunäre Stillwasser-Phase an. Auf die flächenhafte Verbreitung dieser Ablagerungen machte erstmals $\operatorname{KolP}$ (1974: 23, 1975: 20) aufmerksam. In der Nähe des Südlichen Schillgrundes S der Doggerbank im niederländischen Nordseesektor kommt verbreitet eine als „Torfgyttja “ bezeichnete Stillwasser-Ablagerung vor.

Eingeschaltete Hydrobien-Horizonte gehören nach KolP aufgrund von Mollusken-, Foraminiferen-, Ostracoden- und Diatomeen-Bestimmungen in den meso- bis pliohalinen Salinitätsbereich. Der von Behre \& Menke (1969), Pazotka Von Lipinski \& Wiegank (1969) und Diebel \& Pietrzeniuk (1971) sehr detailliert bearbeitete Stechrohrkern 26 von der Station 3 am Südlichen Schillgrund (s. KolP 1974-1976) enthielt in 1,902,80 $\mathrm{m}$ unter Oberfläche (스 44,9-45,8 $\mathrm{m}$ Wassertiefe) tonige Schlicke und Schluffe mit tonigen und humosen Lagen sowie Gyttjabänder, die nach Foraminiferen und Ostracoden im unteren Teil (2,80-2,45/2,35 m unter Oberfläche) dem mio- bis mesohalinen Bereich zuzuordnen sind (s. Abb. 3), während der mittlere Teil (2,45/2,35-2,08 m unter Oberfläche) überwiegend brachyhalin und der obere Teil (2,08-1,90 m unter Oberfläche) vorwiegend euhalin gewesen sein dürfte. Insgesamt sprechen Lithologie und Fauna für eine lagunäre Bildung dieses, der qhKl-Teilsequenz zugeordneten Abschnittes des Kerns 26. 


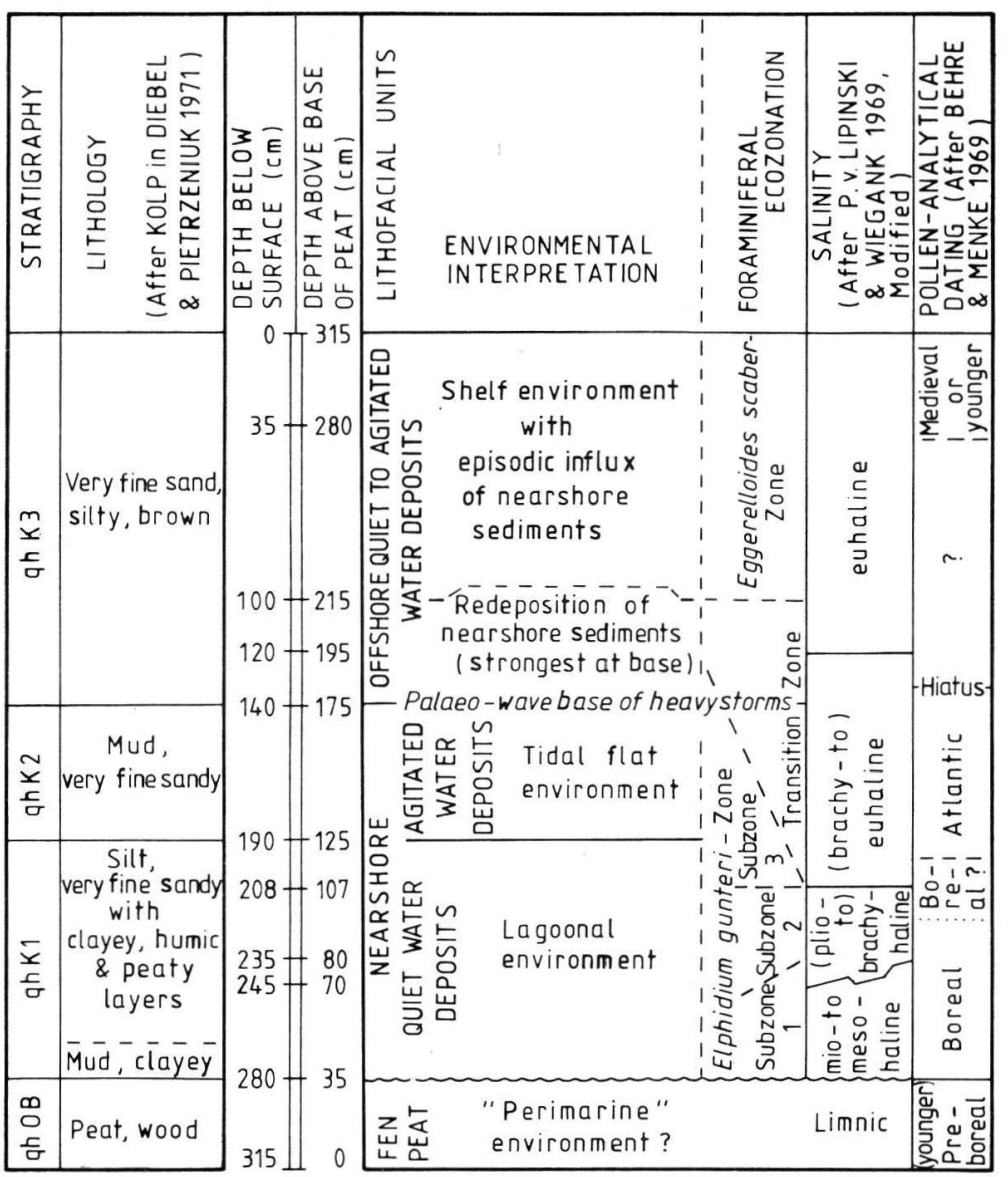

Abb. 3: Gliederung des Kerns 26 der Station 3 (Kolp 1974-1976, Lage s. Abb. 1) nach lithologischen, faziellen und palökologischen Kriterien.

Text-Fig. 3: Division of core 26 of station 3 (Kolp 1974-1976, position s. text-fig. 1) according to lithological, facial and palaeoecological criteria.

Im deutschen Nordseesektor konnte Müller (in Ludwig, Müller \& Streif 1979) in Bg. 235 W Weißer Bank boreale Brackwasser-Tone nachweisen. In den Bg. 280, 196, 172, 137 und 122 sind kalkfreie Tone und Schluffe nach MüLlER nicht-mariner Entstehung. MEYeR (1980) interpretierte gleichartige Schichten in der Bg. 2 V als limnische Flachwasser- bis Ufer-Fazies. In Bg. 284 stufte MülleR einen stark kalkigen Schluff 1,22 m unter Oberfläche (스 $37 \mathrm{~m}$ unter SKN) als nichtmarines IV a (nach Overbeck 1975) ein. Jedoch enthält der höhere Teil der Schlufflage bereits marine Foraminiferen und dürfte damit dem oberen Abschnitt der qhKl-Teilsequenz entsprechen. Das bislang niedrigste Alter dieser Teilsequenz belegte Müller (in Ludwig et al. 1979) mit Pollen der Zone VIII a in Bg. $102 \mathrm{~W}$ vor der N-Spitze von Sylt.

Die tonigen oder schluffigen Ablagerungen der qhKl-Teilsequenz besitzen wie die Organische Basalsequenz nur eine geringe Verbreitung im deutschen Nordseesektor. Sie sind 
bislang nur in Mächtigkeiten $<1 \mathrm{~m}$, meist sogar $<0,5 \mathrm{~m}$ angetroffen worden. Meist lagern küstennahe Bewegtwasser-Ablagerungen (qhK2) unmittelbar auf pleistozänen Decksanden oder auf terrestrischen Sanden des frühen Holozäns.

Gröberklastische nicht-marine Ablagerungen des frühen Holozäns stellen offenbar die kalkfreien bis schwach kalkigen Fein- und Mittelsande im unteren Teil der Bohrungen W und NW Helgoland dar. Über Alter und Genese dieser Sedimente ist noch wenig bekannt. Einen ersten Hinweis auf das Vorhandensein holozäner, terrestrischer Sande lieferte Müller (in Ludwig et al. 1979) mit der Datierung zweier Torf-Lagen in Bg. $234 \mathrm{~W}$ Weißer Bank. Zwischen diesen früh-borealen Torfen (Zone VI a nach Overbeck 1975) lagern kalkfreie Feinsande. Diese Sande belegen, daß während des frühen Boreals Torfbildung und fluviatile (?) und/oder äolische (?) Sedimentation stattgefunden haben.

\subsubsection{Küstennahe Bewegtwasser-Ablagerungen (qhK2)}

Die Ablagerungen der qhK2-Teilsequenz bestehen aus kalkigen Sanden und Schluffen, deren bio- und lithofazielle Merkmale auf Entstehung in einem Milieu mit starker bis sehr starker Wasserbewegung hinweisen. Diese meist poly- bis euhalinen Sedimente gehören den vital-lipostraten und letal-lipostraten Biofazies-Bereichen (SCHÄFER 1963) an; sie werden hier als küstennah-marine Bewegtwasser-Ablagerungen (qhK2) bezeichnet. Die qhK2-Teilsequenz umfaßt alle Sedimente der Watt-, Rinnen-, Strand- und Vorstrandbereiche, die von Wellengang und Gezeiten geprägt worden sind.

In weiten Bereichen der Deutschen Bucht nimmt der Schluffgehalt zum Hangenden der Teilsequenz hin ab. Dementsprechend ist die Schluff- bis Feinsand-Folge - soweit sie abtrennbar ist - meist auf den unteren Abschnitt der Teilsequenz beschränkt (STREIF \& UFFENORDE 1981). In typischen Wechsellagerungen von dunklen, meist grauen Schluffen und helleren Feinsanden sind Linsenschichtung, Wechselschichtung, Übergänge zu Flaserschichtung, Lagen von Torfdetritus und anderen Pflanzenresten sowie bioturbate Horizonte häufig. Der Kalkgehalt ist meist hoch.

Kolp $(1974,1975)$ beschrieb aus entsprechenden Ablagerungen in der Nähe des Südlichen Schillgrundes im niederländischen Nordseesektor einen basalen sandigen CardienHorizont. Im deutschen Nordseesektor kommt ein derartiger Cardien-Horizont nur gelegentlich vor. Cerastoderma edule ist meist unregelmäßig in dieser Teilsequenz verteilt und stellt oft ihr auffälligstes makrofaunistisches Merkmal dar (s. auch SindowsKi 1970:36, Wunderlich 1980: 218). Nach Sindowski entspricht die Molluskenfauna derjenigen der heutigen „Macoma baltica-Gesellschaft".

Küstennahe Ablagerungen der qhK2-Teilsequenz in sandiger Fazies kommen im Gebiet zwischen den Ostfriesischen Inseln und der Doggerbank in weiter Verbreitung vor. Sie bestehen dort meist aus hellbeigegrauen schluffigen Fein- bis Mittelsanden. Nicht selten sind einzelne dunkle Schluff-Bänder und -Lagen sowie Lamellen von Torfdetritus und Pflanzenhäcksel. Flaserschichtung ist meist auf den unteren Teil der küstennahen Sande beschränkt. In diesem Teil wurde auch Kleinrippel-Schichtung verbreitet beobachtet. Mit dem Schluff nimmt auch der Kalkgehalt generell zum Hangenden hin ab. Außer vereinzelten Schillanreicherungen und stark bioturbaten Horizonten sind die Gehalte an Mollusken und Lebensspuren meist gering. Die qhK2-Sande sind sicher polygenetisch. Bei Sanden mit \pm deutlicher Kleinrippel-Schichtung und vereinzelter Flaserschichtung scheint es sich um Sandwatt-Sedimente zu handeln. Sehr gut sortierte Feinsande, die vielfach den Top der Folge bilden, dürften als Wanderfeinsande zu deuten sein (s. STREIF \& UfFENORDE 1980: 6). 
Aus dem deutschen Nordseesektor liegen bislang nur palynologische Datierungen aus den Schluffen der qhK2-Teilsequenz vor. Müller (in Ludwig, Müller \& STReif 1979, 1981 b) wies im ältesten Teil dieses Schichtgliedes Boreal (Zone VI/VII n. Overbeck 1975: Bg. 196, Zone VII: Bg. 172, Zone VII b: Bg. 194) und im jüngsten Teil dieser Einheit hohes Atlantikum (Zone VIII b: Bg. 102, 105, 122) und Subboreal (Zone IX: Bg. 85) nach. Weitere Datierungen ergaben: Boreal (Zone VII: Bg. 168, 210, 245 n. Meyer 1981 a, Bg. 270 n. MEYER 1981 b).

$\mathrm{Da}$ aus der Deutschen Bucht keine Altersangaben aus den qhK2-Sanden selbst, sondern nur einzelne Datierungen aus den unter- und überlagernden Schichten vorliegen, kann ihr Alter nicht sicher angegeben werden. Vermutlich hat sich das Gros dieser Abfolge im höheren Teil des Boreal und im Atlantikum gebildet.

Die größte Mächtigkeit der qhK2-Teilsequenz von $>7 \mathrm{~m}$ wurde in Bg. 13 SindowskI (1970) im „Elbe-Urstromtal“ erbohrt. Die Geestplatten W und E des „Elbe-Urstromtales" enthalten küstennahe Ablagerungen meist $<3$ m mächtig.

2.2.3. Küstenferne Stillwasser-bis Bewegtwasser-Ablagerungen (qhK3)

Die qhK3-Teilsequenz enthält Sedimente, die nach bio- und lithofaziellen Merkmalen auf dem offenen Schelf unterhalb der Wellenbasis entstanden sind und nur durch Strömungen und Stürme beeinflußt wurden. Die Biofazies entspricht vorwiegend dem vitallipostraten, untergeordnet dem vital-pantostraten Bereich (SCḦ̈FER 1963).

Küstenfern-marine Sande und siltige Sande bilden in weiter Verbreitung die jüngste Teilsequenz der holozänen Klastischen Sequenz. Sie überlagern in der Deutschen Bucht meist mit \pm ausgeprägter Erosionsdiskordanz, verschiedene der oben genannten stratigraphischen und faziellen Einheiten (vgl. JANSEN, van WeERING \& Eisma 1979, WunderLICH 1980).

So liegt diese Teilsequenz z. B. in den Bg. 2 V, 235 und 284 unmittelbar auf nichtmarinem Ton und Silt, in Bg. 238, 3 V, 133, 130, 125, 119 und 117 auf nicht-marinem Fein- und Mittelsand, in den Bg. 234, 287 und 146 auf Basaltorf und in den Bg. 351, 335, 280 und 137 auf küstennahem Silt und Feinsand. Auf den Arealen mit hochliegendem Pleistozän am Ostrand des „Elbe-Urstromtales“ (FIGGE 1980), W der Nordfriesischen Inseln und im Gebiet des Borkumer Riffgrundes lagern küstenfern-marine Ablagerungen stellenweise transgressiv auf Sedimenten des Pleistozäns und Pliozäns. Nach eigenen Untersuchungen gehören dazu Mittelsande mit basaler Kieslage, die in Bg. 219 Geschiebemergel überlagern. In vielen Bohrungen zeigen Schill-Anreicherungen und/oder Korngrößenzunahme an der Basis, unregelmäßiger Verlauf der Grenzfläche, deutlicher Material- und Farbwechsel, teilweise auch Grabgänge an, daß an der Untergrenze dieser Teilsequenz eine Schichtlücke, gelegentlich vielleicht auch ein stratigraphischer Kondensationshorizont in Form eines "lag-deposit" liegt. Bohrungen mit diesem Schill-Horizont zeigen makroskopisch bereits an der Zusammensetzung der Mollusken-Fauna, mikroskopisch an der Zusammensetzung der Foraminiferen-Fauna den \pm starken Anteil küstennahen Materials. Auch die sedimentologischen Merkmale weisen auf eine Erosionsdiskordanz hin (vgl. Wunderlich 1980).

Uber diesem basalen Teil herrschen dunkelolivgrüne, grünlichgraue und -braune, stark bioturbate, meist kalkreiche, \pm schluffige Feinsande vor, die generell zum Hangenden und zur Küste hin sandiger, z. T. mittelsandig, und bräunlich-hellbeige werden. In vielen Bohrungen ist eine $Z_{w}$ eiteilung dieser feinklastischen Abfolge in einen schluffigen unteren 
und einen sandigen oder lagenweise sandigen oberen Abschnitt möglich. Der Schill-Gehalt nimmt im unteren Abschnitt von unten nach oben ab, im oberen Abschnitt wieder etwas $\mathrm{zu}$.

Wie Reineck, Dörjes, Gadow \& Hertweck (1968), Gadow \& Reineck (1969) und REINECK \& SINGH (1972) nachgewiesen haben, sind die Sandlagen in den stärker siltig entwickelten Schlickgebieten südlich Helgoland Sturmschichten. Dies ist nicht nur sedimentologisch, sondern auch faunistisch durch allochthone Lagen mit der Wattschnecke Peringia ulvae, Fluchtspuren, autochthone bis parautochthone Schill-Lagen u. s. w. zu belegen.

Bio- bzw. ökostratigraphisch ist die qhK3-Teilsequenz durch das Auftreten von Mollusken wie Turritella communis, Venus (Chamelea) striatula (气 Venus gallina), Corbula gibba, Cultellus pellucidus, Abra alba, Nucula nitida, Acanthocardia echinata (스 Cardium echinatum) und Thyasira sarsi gekennzeichnet (s. HERTWECK \& REINECK 1969 mit erster Artenübersicht aus dem Elbe-Urstromtal NW Helgoland). Diese Arten kommen vielfach parautochthon in Schill-Lagen in wechselndem Mengenverhältnis vor. Von diesen Schill-Lagen ist der Schill-Horizont an der Basis der qhK3-Teilsequenz am mächtigsten und am weitesten verbreitet. Je nach Zusammensetzung kann er als Turritellen-Horizont, Veneriden-Horizont u. s. w. ausgebildet sein.

Kolp (1974-1976) beschrieb den Turritellen-Horizont aus einem Profil in der Nähe des Südlichen Schillgrundes. Auf einer 1981 mit dem F. S. „GAuss“ durchgeführten Bohrkampagne wurde dieser Turritellen-Horizont zwischen Borkum Riffgrund und Nördlichem Schillgrund verbreitet angetroffen und der Übergang in einen Veneriden-Horizont beobachtet.

Im unteren Teil dieses basalen Schill-Horizontes sind vielfach Mollusken angereichert, die aus den unterlagernden Schichten aufgearbeitet und umgelagert wurden (z. B. auf-

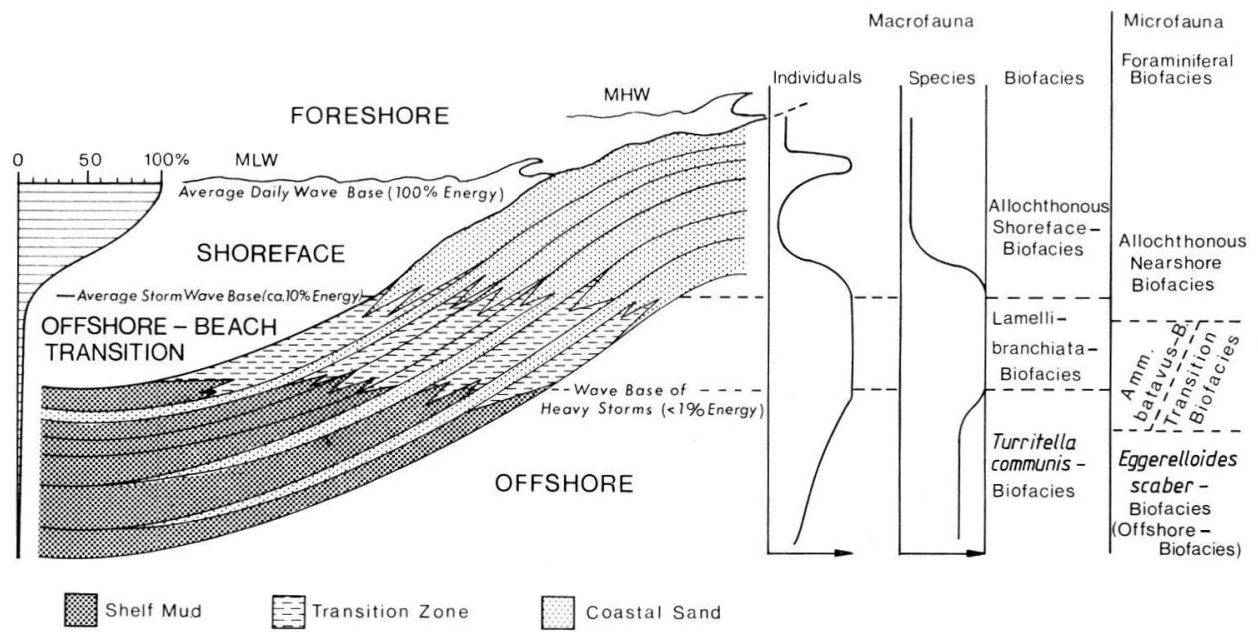

Abb. 4: Küste-Schelf-Modell nach ReInECK \& SingH (1980). Biofazies-Gliederungen ergänzt: Biofazies-Einheiten für die Makrofauna übertragen aus HERTwECK (1971). Foraminiferen-Biofazien nach eigenen Untersuchungen. Anstelle des Schelfschlickes können Relikt-Sedimente mit anderer Faunenzusammensetzung vorkommen.

Text-Fig. 4: Beach-shelf modell after REINECK \& SingH (1980). Biofacial divisions added: macrofaunal biofacies units adopted from HerTwECK (1971). Foraminiferal biofacies after own investigations. Relictsediments with different faunal composition can occur instead of shelf mud. 
fällig rostfarbene Cardien in Bg. 1715). Zu den Formen, die mit abnehmender Häufigkeit zum Hangenden hin vorkommen, gehören Cerastoderma edule, Macoma baltica, Mytilus edulis, Peringia ulvae, Hydrobia neglecta und ventrosa.

Entsprechend rezenten ökologischen Befunden von HeRTwECK (1971 a), DöRJES \& Hertweck (1975), Reineck \& Singh (1973/1980) kann ein Maximum in Lamellibranchiata-Arten und -Individuen als Übergangsbereich zwischen Schelf und Küste („offshorebeach transition" REINECK \& SINGH 1980: 423, Fig. 583; s. Abb. 4) bzw. Mittleres Sublitoral gedeutet werden. In diesem Bereich sind Einflüsse des Seeganges relativ unbedeutend und beschränken sich meist auf Zeitabschnitte, in denen schwere Stürme die Wellenbasis absenken und großflächig Erosions- und Resedimentationsvorgänge verursachen. In Küstensand-Schelfschlick-Profilen folgt unter den Macoma- und Venus-Coenosen die Amphiura-Coenose. Letztere bleibt weitgehend auf den Übergangsbereich bzw. das Mittlere Sublitoral beschränkt. In Silt-Gebieten schließt sich die Turritella communisCoenose zum Unteren Sublitoral hin an.

Diese biozönotische Gliederung ist nur mit Einschränkungen auf Paläomilieus übertragbar. Bereits HeRTwECK (1971 b) hatte Schwierigkeiten, aus der klaren, von DörJes (1971) erarbeiteten Biocoenosen-Abfolge eine Biofazies-Gliederung zu rekonstruieren. Aufgrund häufiger Sedimentumlagerung im Vorstrand-Bereich schlug er vor, „nur ganz allgemein von einer "Allochthonen Vorstrand-Biofazies" zu sprechen" (S. 272). Da Schlangensterne anhand ihrer einzelnen Skelett-Elemente nicht mehr bestimmt werden können, d. h. als fazielle Leitfossilien demnach wertlos sind, bezeichnete HeRTwECK den Faziesbereich der Amphiura-Coenose als "Lamellibranchiaten-Biofazies des oberen Schelfbereiches" wegen des in dieser Biofazies auftretenden Maximums an Mollusken-Arten. Lediglich die Turritella communis-Coenose dürfte auch fossil, d. h. als Biofazies erhaltungsfähig sein.

Die oben erwähnten Schill-Horizonte an der Basis der qhK3-Teilsequenz enthalten somit umgelagertes Material entweder aus der Lamellibranchiaten-Biofazies und der allochthonen Vorstrand-Biofazies oder aus Turritellen-, Lamellibranchiaten- und allochthonen Vorstrand-Biofazies, wobei das Material der jeweils am tiefsten gelegenen Biofazies weitgehend als (par-)autochthon angesehen werden kann.

Pollendatierungen aus der qhK3-Teilsequenz liegen z. Zt. nur von 4 Bohrungen vor. Die ältesten Datierungen lieferte Bg. 208 mit Einstufungen in Zone VII und VII-VIII a nach Overbeck (Ludwig, Müller \& Streif 1979). Die Probenteufe ist nach Seekarte wahrscheinlich $5 \mathrm{~m}$ tiefer als angegeben: 45,0-45,1 $\mathrm{m}$ und 43,8-43,9 $\mathrm{m}$ unter SKN. Müller (1981 a) konnte die Basis der qhK3-Teilsequenz der Bg. 133 als Zone X nach Overbeck datieren (40,8-40,9 m unter SKN). Zwei dicht unter der Oberfläche entnommenen Proben aus Bg. 213 und 214 ergaben Zone XII.

Die vorliegenden Bohrungen zeigen nur im Bereich des Elbe-Urstromtales qhK3Mächtigkeiten von $>5 \mathrm{~m}$. Auf den ehemaligen Hochflächen W und E dieses Urstromtales ist die Mächtigkeit dieser Teilsequenz meist $<2 \mathrm{~m}$.

\subsubsection{Zur Gliederung der Klastischen Sequenz mit Foraminiferen}

Durch Untersuchung der Foraminiferen-Fauna wurde versucht, die oben beschriebene Gliederung des Nordsee-Holozäns biofaziell zu präzisieren. Dies ist mittels quantitativer Analysen möglich. Deshalb wurden die Foraminiferen durch Schweretrennung mit $\mathrm{CC}_{4}$ angereichert und je Probe nach Möglichkeit > 300 Exemplare bestimmt und gezählt. Wie die Beispiele in Abb. 5-8 zeigen, ist eine recht deutliche Foraminiferen-Zonierung zu 
erkennen, die zusammen mit anderen biotischen Faktoren (z. B. Schillgehalt, Bioturbation) und abiotischen Faktoren (z. B. Kalkgehalt, Korngrößenverteilung, Sedimentgefüge) eine relativ gesicherte Interpretation des Ablagerungsmilieus und der Veränderung des Milieus im Laufe der holozänen Transgression in den einzelnen Bohrungen erlaubt. Dabei ist bislang gute Übereinstimmung mit dem von Reineck, DörJes, Gadow \& HerTweck (1968), Reineck \& Singh (1973/1980) u. a. aus der Verteilung der Sedimente, der Sedimentgefüge und der Fauna in der Deutschen Bucht abgeleiteten Küste-Schelf-Modell zu beobachten (s. Abb. 4). Die für diesen Faziesraum existierende, jedoch nur auf wenigen Proben basierende Darstellung von HAAKe (1962) und Richter (1967) über die Foraminiferen-Verteilung nördlich der Ostfriesischen Inseln kann ebenfalls für die Gliederung der HolozänProfile der südlichen Nordsee angewandt werden. Richter (1967: 308) bezeichnete den Bereich „stark durchströmter Rinnen und wandernder Sandriffe vor der Küste des inneren Teils der Deutschen Bucht" bezüglich ihrer Foraminiferen-Gemeinschaften als "tote Zone". Daran schließen die Foraminiferen-Gemeinschaften der offenen See an, die auch Elphidium excavatum selseyense, Ammonia batavus ( $\hat{=}$ "Streblus beccarii“), Eggerelloides scaber (스 „Eggerella scabra"), Fursenkoina fusiformis (스 „Virgulina fusiformis") und einer artenreichen, aber individuenarmen kleinen Kalk- und Sandschaler-Fauna besteht.

Die im offshore-Holozän offenbar weniger verbreiteten Ablagerungen des Wattbereiches und des bislang noch nicht nachgewiesenen Groden-Bereiches sind aktuopaläontologisch eingehend bearbeitet worden (HAAKE 1962, Richter 1961, 1964 a, 1964 b, 1965, $1967 \mathrm{im}$ ostfriesischen Küstengebiet). Foraminiferen-Faunen dieser Milieus erweisen sich in jüngster Zeit zunehmend relevant für die Bestimmung holozäner Meeresspiegelstände und deren benachbarter Paläo-Milieus (s. Culver \& Banner 1978, ScotT \& Medioli 1978, 1980).

Richter (1967: 317) unterschied folgende Watt-Biotope nach Foraminiferen-Taphozönosen: (1) brackische Grodengewässer mit agglutinierten Brackwasser-Anzeigern, (2) hohe Watten mit brackischem Einfluß mit Elpbidium gunteri und Miliammina fusca, (3) hohe, geschützte Watten ohne brackischen Einfluß mit Elphidium williamsoni ( $\triangleq$ „E. excavatum"), (4) offene Watten in Turbulenzbereich ohne typische Arten, aber mit Elphidium excavatum selseyense, Ammonia batavus ( $\hat{=}$ "Streblus beccarii“) und marinen Formen als häufigere Arten, (5) stark durchströmte Rinnen und Sandplatten mit Ammonia batavus ( $\hat{=}$ „Streblus beccarii").

Am Beispiel des Kerns 26 der Station 3 in der Nähe des Südlichen Schillgrundes (niederländischer Nordseesektor), dessen Foraminiferen-Fauna von PAzOTKA von LipiNski \& WIEGANK (1969) sehr detailliert analysiert worden ist, soll zunächst überprüft werden, ob und inwiefern die Foraminiferen-Verteilung die oben dargelegte fazielle Gliederung des klastischen Holozäns widerspiegelt.

Abb. 3 zeigt die aus der lithologischen Beschreibung (n. Kolp in Diebel \& PietrzeNIUK 1971) abgeleitete lithostratigraphische und lithofazielle Gliederung im Vergleich zu der aus der Foraminiferen-Verteilung abgeleiteten Salinitätsbestimmung und palökologischen Zonierung. Die Milieu-Interpretation stellt die zusammenfassende Deutung der Befunde von Pazotka von Lipinski \& Wiegank (1969), Behre \& Menke (1969) und Diebel \& Pietrzeniuk (1971) durch den Verfasser dar.

Die 2,80 m mächtige Klastische Sequenz kann nach PazotKa von Lipinski \& WiEgank in 4 Abschnitte gegliedert werden (Klassifikation der Salzgehalte nach Hiltermann 1966:

I. Mio- bis mesohaliner Bereich (2,80-2,45/2,35 m unter Oberfläche):

Charakteristisch für diesen Bereich ist eine artenarme Foraminiferen-Fauna mit Ammonia flevensis, Elphidium gunteri, E. asklundi und Nonion germanicum ( $\triangleq$ „N. depressulum").

Dieser Abschnitt wird hier als Subzone 1 der Elphidium gunteri-Okozone bezeichnet. 
II. Plio- bis brachyhaliner Bereich (2,45/2,35-2,08 m unter Oberfläche):

Neu hinzutretende Formen in diesem Bereich sind Buccella frigida „ssp. 1“, Bolivina pseudoplicata, Bulimina spp., Buliminella elegantissima, Cassidulina crassa, Cibicides lobatulus, Elphidium gerthi, E. williamsoni (今 „E. excavatum"), Nonion depressulum ( $\hat{=}$ „N. umbilicatum") und Quinqueloculina seminulum. Ammonia beccarii, Elphidium excavatum selseyense und Nonion germanicum kommen häufig vor.

Die Mehrzahl der zuerst genannten kleinen Formen scheint allochthon zu sein. Nach der Vielzahl dieser Formen zu urteilen, scheint das Milieu bereits vorwiegend brachyhalin, d. h. marin beeinflußt gewesen zu sein. Die Untergrenze dieses hier als Subzone 2 der Elpbidium gunteri-OKkozone bezeichneten Bereiches ist nicht so scharf begrenzt wie von Pazotka von Lipinski \& Wiegank angegeben.

III. (Brachy- bis) euhaliner Bereich (2,08-1,20 m unter Oberfläche):

In diesem Abschnitt treten verbreitet Ammonia batavus, Bulimina spp., Buliminella elegantissima, Elphidium williamsoni, E. magellanicum, Fissurina spp. und Nonion depressulum sowie vereinzelt Lagena spp. auf. Aubignyna perlucida (스 „Ammonia? perlucida"), Elphidium gerthi, E. incertum und Virgulina fusiformis sind lagenweise häufig. Diversität und Abundanz liegen lagenweise sehr hoch. Im Gegensatz dazu sind Ammonia beccarii und Elphidium excavatum selseyense kontinuierlich sehr häufig. Ebenfalls sehr stetig ist das Vorkommen von Ammonia flevensis, Buccella frigida „ssp. 1“, Bulimina elongata, Cassidulina crassa, Elpbidium asklundi, E. gunteri, Nonion germanicum, $N$. depressulum und Quinqueloculina seminulum.

Dieser Teil ist nach Foraminiferen bereits stark euhalin geprägt. Er kann - je nach Betonung der (par-)autochthonen, in der Mehrzahl großen und stetigen Faunenelemente oder der allochthonen, in der Mehrzahl kleinen und unstetigen Faunenelemente - als Subzone 3 der Elphidium gunteri-Ókozone oder der Ubergangszone (zur Eggerelloides scaber-OOkozone) zugeordnet werden.

IV. Euhaliner Bereich (1,20-0 m unter Oberfläche):

Die Basis dieses Abschnittes ist gekennzeichnet durch eine deutliche Verringerung der Individuenzahl von Elphidium williamsoni und E. gunteri bei gleichzeitiger Häufigkeitszunahme von Asterigerinata mamilla (气 „Asterigerina sp."). In Teufen $\langle 1,00 \mathrm{~m}$ unter Oberfläche wird Eggerelloides scaber (스 „Eggerella scabra“) häufig. Der Abschnitt wird hier unterhalb 1,00 m unter Oberfläche als Ubergangszone, oberhalb dieser Teufe als Eggerelloides scaber-Ökozone bezeichnet. Er ist nach PazotKA von Lipinski \& WieganK (1969: 169) „durch rhythmische Sedimentation und eine rhythmische Schwankung der Individuenzahl fast aller Foraminiferen-Arten gekennzeichnet".

Wie Abb. 3 zeigt, stimmen weder die auf den Untersuchungen von PAzotKa von LIPINSKI \& WIEGANK basierenden ökostratigraphischen Zonen noch die Grenzen, die nach den pollenanalytischen Datierungen von BEHRE \& MENKE (1969) gezogen werden können, mit der lithostratigraphischen Gliederung überein. Eine Erklärung für diesen Befund liegt möglicherweise in der vom Liegenden zum Hangenden zunehmenden diskontinuierlichen Zufuhr von allochthonem Sedimentmaterial. Dieses Material enthält vorwiegend Formen der unmittelbar benachbarten Ókozonen. So enthielt z. B. die 1,35-1,40 m unter Oberfläche entnommene Probe zwar noch die charakteristische Foraminiferen-Fauna der Elphidium gunteri-Ökozone, ist jedoch lithostratigraphisch eindeutig den küstenfernen Bewegtwasser-Ablagerungen (qhK3) zuzurechnen. Wie bei den Foraminiferen, so schließt auch die pollenanalytische Datierung dieser Probe in das Atlantikum diesen Teufenbereich ohne Hiatus an die ebenfalls dem Atlantikum zugeordneten küstennahen BewegtwasserAblagerungen im Liegenden an. 
Hingegen unterstützt die Zusammensetzung der Ostracoden-Fauna (s. Diebel \& Pietrzeniuk 1971) die lithostratigraphische Zuordnung zur qhK3-Teilsequenz. So zeigt das verbreitete Auftreten von Pterygocythereis jonesi an, daß die Sedimentation unterhalb der Sturmwellen-Basis im küstenfern-marinen Milieu erfolgte (vgl. Liebau 1980). Die im Watt-Milieu noch recht verbreiteten Arten Cyprideis torosa und Cytheromorpha fuscata sind verschwunden. Diebel \& Pietrzeniuk (1971) erkannten zwar den Charakter des Teufenabschnittes zwischen 2,08 und $1,52 \mathrm{~m}$ unter Oberfläche $(\hat{=} 1,07-1,63 \mathrm{~m}$ über Torfbasis) als einen palökologischen Überlappungsbereich; die von ihnen ermittelte Salinität des Ablagerungsmilieus in diesem Bereich als pliohalin bis brachyhalin ist jedoch zweifellos zu niedrig. Elofsonella concinna und Pterygocythereis jonesi sprechen für brachyhaline bis euhaline Salzgehalte dieses Übergangsbereiches. Im höheren Teil des Profiles verhindert die Seltenheit von Ostracoden gesicherte palökologische Angaben. Hier läßt sich bei den wesentlich häufigeren Foraminiferen mit dem ersten verbreiteten Vorkommen von Eggerelloides scaber eine deutliche Grenze zwischen Ubergangszone und scaber-Ökozone ziehen. In dieser Ökozone wird das Schelf-Milieu endgültig erreicht.

Der Kern 26 zeigt sehr anschaulich, daß die Foraminiferen zwar sehr gut zur Bestimmung der Paläomilieus in der holozänen Klastischen Sequenz geeignet sind, daß sie jedoch durch Verdriftung und Verfrachtung in benachbarte Milieus gegenüber den lithofaziellen Wechseln etwas versetzte Grenzen oder Übergangsbereiche aufweisen.

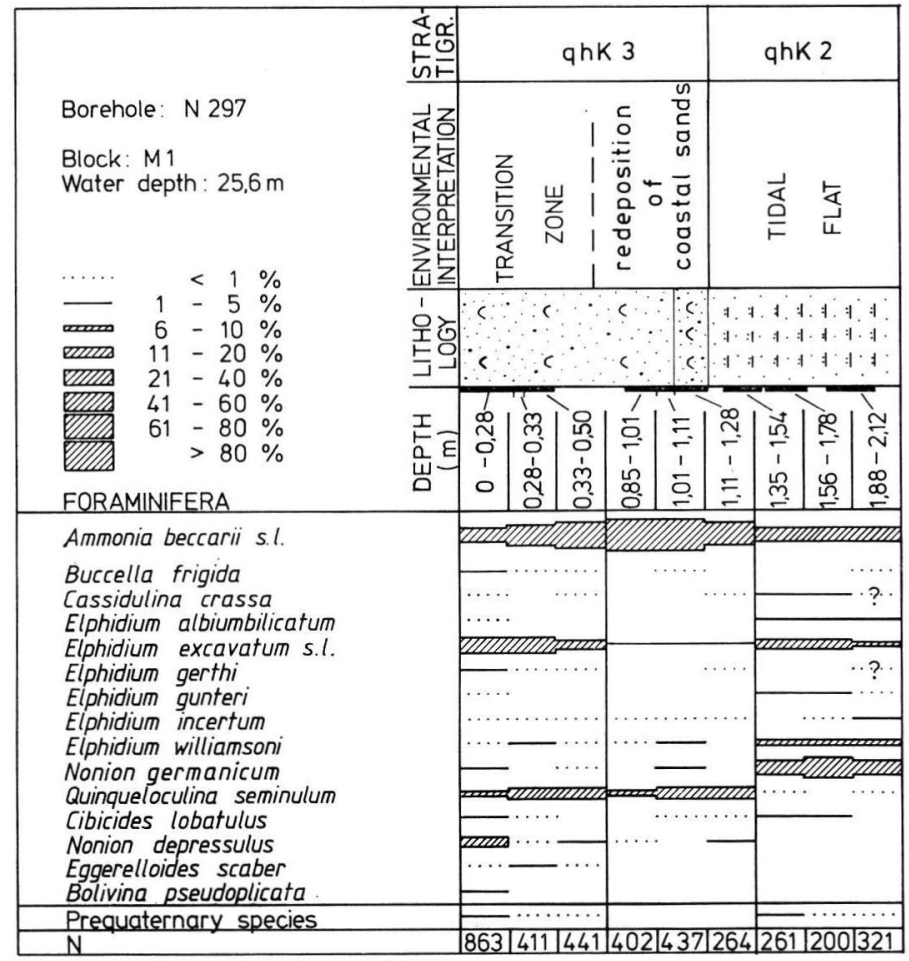

Abb. 5: Palökologische Gliederung und Interpretation der Bohrung 297 mit Foraminiferen. Dargestellt wird die Verbreitung der wichtigsten Arten mit $>1 \%$-Anteil an der ForaminiferenFauna mindestens 1 Probe. $\mathrm{N}=$ Stichprobengröße.

Text-Fig. 5: Palaeoecological division and interpretation of borehole 297 with foraminifera. Only those important species are figured, which have a percentage of $1 \%$ or more of the foraminiferal fauna in at least 1 sample. $\mathrm{N}=$ size of random sample. 
Bg. 297 (Abb. 5) enthält 3 Foraminiferen-Ökozonen, die in Verbindung mit lithologischen und makrofaunistischen Befunden 2 Fazieseinheiten erkennen lassen. Die Proben 1,35-1,78 m unter Oberfläche werden der Elphidium excavatum-Ökozone zugeordnet. Ihre Interpretation als Watt-Ablagerungen steht in Übereinstimmung mit lithologischen Merkmalen wie rhythmischem Wechsel von Feinsand und Schluff mit ausgeprägten Linsenund Flaserschichtungsgefügen und hohem Kalkgehalt. Mit scharfer Grenze lagern darüber zunächst stark schillhaltige, schlecht sortierte Fein- bis Mittelsande, dann zum Hangenden abnehmend schillführende, sehr gut sortierte Feinsande. Sie führen in den Proben 0,85$1,28 \mathrm{~m}$ unter Oberfläche eine individuenreiche, aber artenarme Fauna mit stark skulptierten, großwüchsigen Ammonia-Formen und große Quinqueloculinen der Ókozone der „Abroll-Foraminiferen“. Die Proben aus $0-0,50 \mathrm{~m}$ unter Oberfläche enthalten eine Foraminiferen-Fauna der Übergangszone mit Eggerelloides scaber $\langle 5 \%$.

Im Gegensatz zur Foraminiferen-Fauna, die von küstennah-marinen Faunenelementen insbesondere des Küstensandes geprägt ist, unterstützt die Makrofauna die lithostrathigraphische Zugehörigkeit des Abschnittes 0,85-1,28 m unter Oberfläche zur qhK3-Teilsequenz. Die Zugehörigkeit des schillreichen Fein- bis Mittelsandes im Hangenden der WattAblagerungen zur qhK3-Teilsequenz wird bereits makrofaunistisch durch die Zusammensetzung der Mollusken-Fauna bestätigt (Venus [Chamelea] striatula, Corbula gibba, Nucula nitida, Lunatia nitida; Fehlen von Cerastoderma edule, Macoma baltica und Mytilus edulis).

Bg. H $15-3 \mathrm{~V}$ (Abb. 6) besitzt im Hangenden eines schillfreien, wahrscheinlich pleistozänen Feinsandes mit einzelnen präquartären Foraminiferen eine in 5 lithologische Einheiten gliederbare holozäne Klastische Sequenz. Zwischen dem basalen, schillreichen Feinsand und einem 0,12 m mächtigen feinsandigen Schill schaltet sich eine 0,04 m mächtige Torfdetritus-Lage ein. Die darüber lagernden Feinsande sind im unteren Teil stark schillhaltig; im höheren Teil nimmt der Schillgehalt stark ab. Hier kommen nur juvenile Lamellibranchiaten vor. Die obersten $0,66 \mathrm{~m}$ mächtigen Torfdetritus-führenden Feinsande enthalten zwar ebenfalls sehr wenig Mollusken, aber wieder einzelne adulte Lamellibranchiaten.

Nach Foraminiferen gehören die schillreichen Partien in die Übergangszone, wobei im unteren Teil mit hohen Prozentanteilen von Ammonia batavus ( $>40 \%$ ) und Elpbidium williamsoni $(>10 \%$ ) noch ein starker Einfluß küstennaher Milieus zu beobachten ist, der bis zum Horizont mit juvenilen Lamillibranchiaten allmählich abnimmt. Mit diesem Horizont beginnt die Eggerelloides scaber-Ökozone. Deutlich nimmt auch die Individuenzahl von Fursenkoina fusiformis zu. In Übereinstimmung mit den aktuopaläontologischen Befunden von RichteR (1967) wird angenommen, daß mit diesem Horizont das küstenferne Milieu der offenen Nordsee bzw. des Schelfes erreicht wurde.

Da außer Turritella communis alle biofaziell wichtigen Mollusken der qhK3-Teilsequenz bereits in den Basisschillen vorkommen (Venus [Chamelea] striatula, Corbula gibba, Cultellus pellucidus, Nucula nitida, Thyasira sarsi u. a.), ist die gesamte holozäne Schichtenfolge dieser Teilsequenz zuzuordnen.

In Bg. 350 (Abb. 7) lagern gut bis sehr gut sortierte Feinsande der qhK3-Teilsequenz ohne basale Schillanreicherung auf Foraminiferen-freien terrestrischen Tonen des Pleistozäns oder frühen Holozäns. Durch Eggerelloides scaber $(>20 \%)$ und Fursenkoina fusiformis $(>5 \%$ ) lassen sich auch in den Feinsanden dieser Bohrung die Schelf-Ablagerungen von denen der Übergangszone abtrennen. Bg. 114 (Abb. 8) zeigt zwei ausgeprägte Häufigkeitsmaxima von Eggerelloides scaber. Das obere Maximum aus bioturbaten, schwach tonig/schluffigen, feinsandigen Mittelsanden entspricht wie in Bg. 350 dem rezenten Siedlungsmilieu dieser Art. In Übereinstimmung mit diesem Befund gibt Richter (1967: 310, 


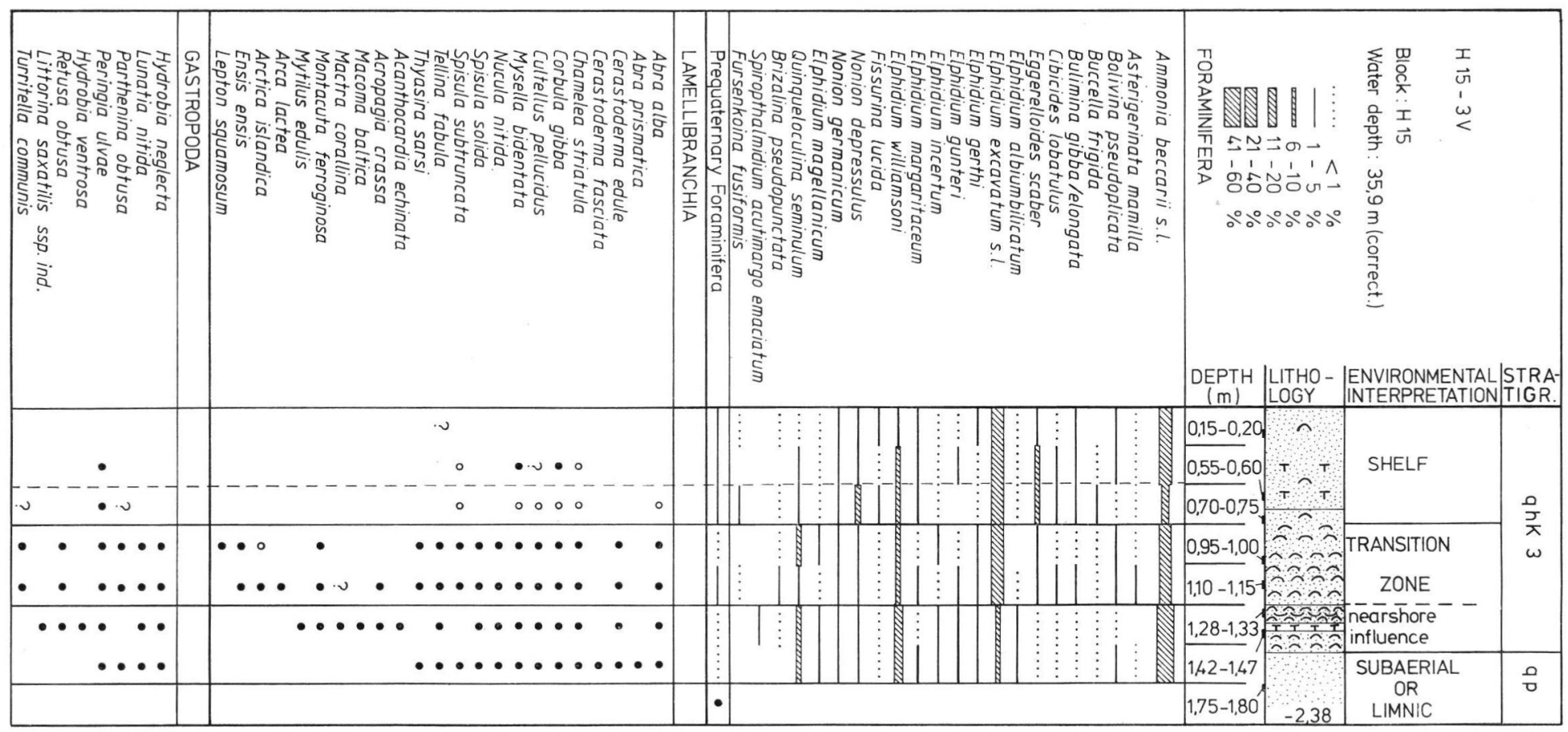

Abb. 6: Palökologische Gliederung und Interpretation der Bohrung $\mathrm{H} 15-3 \mathrm{~V}$ mit Foraminiferen und Mollusken ( $\mathrm{O}=$ juvenile Exemplare). Wassertiefe auf SKN korrigiert.

Text-Fig. 6: Palaeoecological division and interpretation of borehole $\mathrm{H} 15-3 \mathrm{~V}$ with foraminifera and molluscs $(0=$ juvenile specimens). Water depth corrected to - SKN (German Admiralty Datum which corresponds to mean spring low tide). 


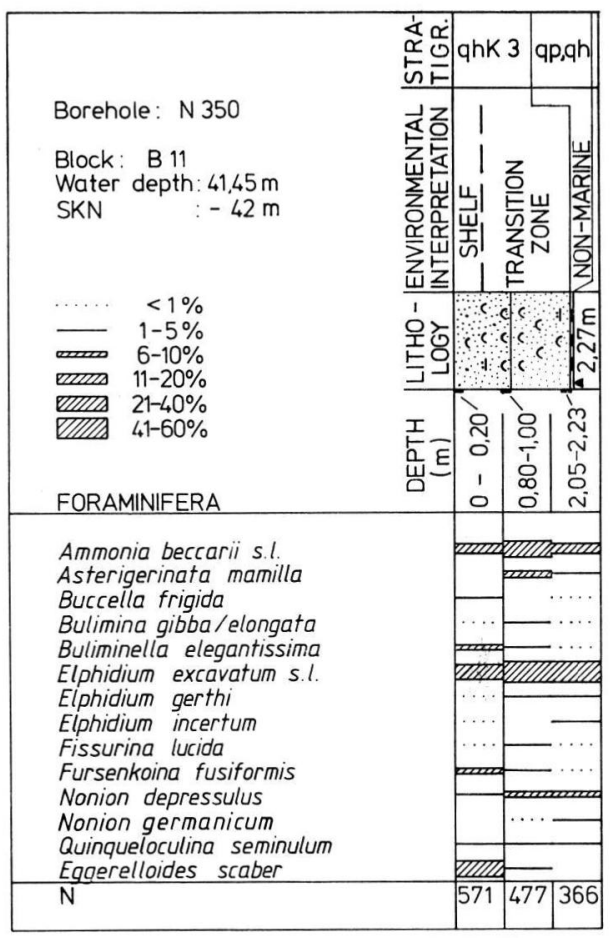

Abb. 7: Palökologische Gliederung und Interpretation der Bohrung 350 mit Foraminiferen.

Text-Fig. 7: Palaeoecological divison and interpretation of borehole 350 with foraminifera.

Abb. 9 E-F) für die Probenserien von 2 Stationen unweit der Bg. 114 aus 33-36 m Wassertiefe Prozentanteile dieser Art an der Lebendfauna von 18,5-39,0\% und an der Totfauna von 16,2-19,2\% an. Bereits JARKE (1961: 27) erkannte, daß die „Eggerella-Provinz" vor allem in den etwas tieferen Gebieten der heutigen Nordsee anzutreffen sei. Das untere Maximum besitzt zwar die gleiche Korngrößenverteilung wie das obere, ist aber Mittelsand-streifig. Dieser scaber-Horizont ist möglicherweise mit dem unteren Teil der scaber-Zone in Bg. H $15-3 \mathrm{~V}$ zu parallelisieren. Weitere Funde dieses Teils der scaberZone in den Bg. H $15-2$ V, H $15-4$ V, 1705, 1706, 1708, 1709, 1715, 1718, 1725, 1727, 1730 scheinen die Vermutung zu bestätigen, daß es sich um einen weit verbreiteten Horizont handelt. Möglicherweise bestehen überregionale, ökostratigraphische Bezüge zum Ostsee-Holozän („Eggerella-Zone“, Lutze 1965, 1974; Exon 1972; Winn 1974; Wefer, WEBER \& ERLENKEUSER 1978).

Nach Exon (1972: 32) überlagern in der Flensburger Außenförde die Eggerella-reichen Schichten brackische Sedimente des Subboreals, deren Alter mit $3000-2000$ v. Chr. angegeben werden. Diese Schichten umfassen nach Averdieck (1972) den höheren Teil der Pollenzone IX bis XI (unterer Teil) nach Overbeck, d. h. höheres Subboreal und tieferes Subatlantikum. Lutze (1974) gab für die Eggerella-Zone eine Zeitspanne von ca. 2800 Jahren an (ca. 1000 v. Chr. -1800 n. Chr.).

Exon (1972) führt das häufigere Auftreten von Eggerella scabra auf Zunahme der Westwinde (subatlantisches Klima) vor ca. 3000 v.h. zurück, die einen stärker marinen 


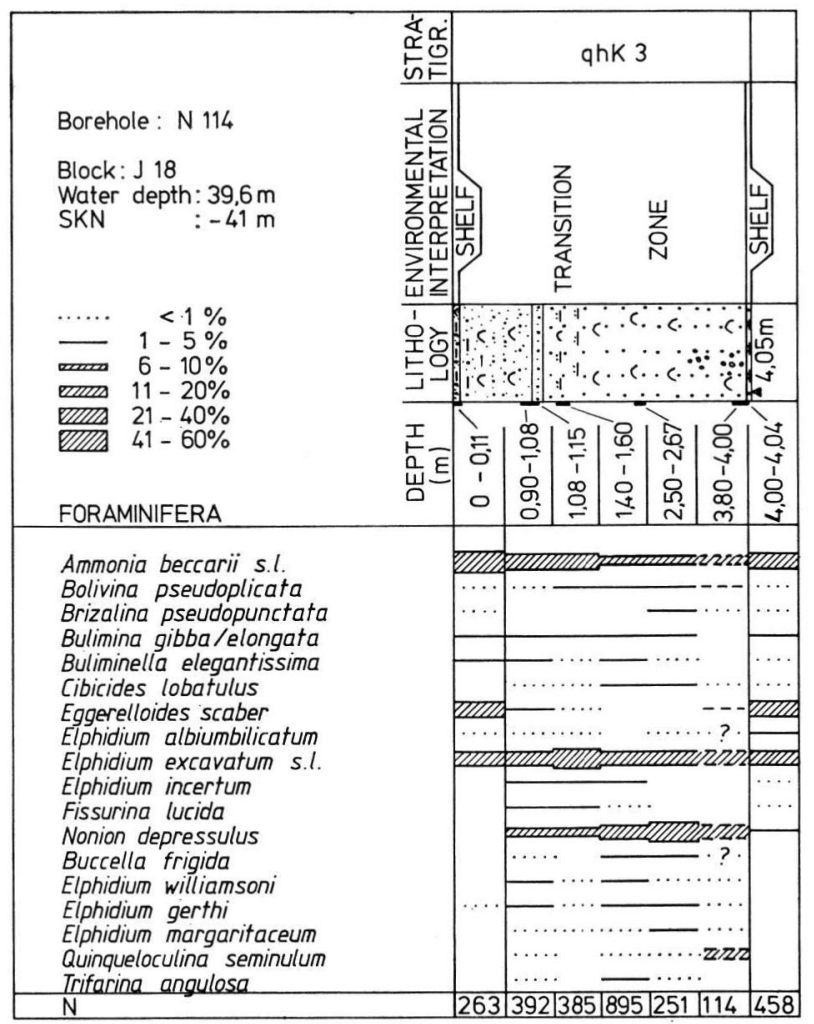

Abb. 8: Palökologische Gliederung und Interpretation der Bohrung 114 mit Foraminiferen.

Text-Fig. 8: Palaeoecological division and interpretation of borehole 114 with foraminifera.

Einfluß in der Ostsee verursacht haben sollen. Jedoch zeigen die Verteilungsdiagramme von Kastenlot-Kern 10872 (Exon 1972: Fig. 10), daß der größte Teil der Eggerella-Zone im Subboreal liegt. Der Klimawechsel an der Wende zum Subatlantikum mit Zunahme der Westwinde scheint vielmehr zusammen mit stärkerer Einfrachtung von Sand und Kotpillen auch eine Umlagerung der Eggerelloiden verursacht zu haben. Diese Umdeutung der Verteilungsdiagramme von ExoN wird unterstützt durch die Beobachtungen von WINN (1974), wonach die schlickigen, stark bioturbaten Sande seiner Einheit F mit hohen Prozentanteilen von Eggerella scabra durch Erosionsvorgänge an der Basis der obersten Deckschicht (Einheit $G$ ) vielfach völlig aufgearbeitet und offenbar in diese Deckschicht eingefrachtet wurden.

Der küstenferne Fein- und Mittelsand der in Kap. 2.2.3 beschriebenen qhK3-Teilsequenz weist bemerkenswerte Parallelentwicklungen zu der WinN'schen Gliederung auf (s. auch WefER et al. 1978). Liegt eine Dreiteilung dieses Schichtgliedes - Basisschill nicht mitgerechnet - vor, so enthält der feinkörnige, stärker bioturbate mittlere Teil neben Resten und Spuren von Echinocardien höhere Prozentanteile von Eggerelloides scaber und ein Minimum von Ammonia batavus (s. Bg. $\mathrm{H} 15-3 \mathrm{~V}, \mathrm{Abb}$. 6). In dieser Boh- 
rung ist der noch bestehende höhere Eggerelloiden-Anteil in Probe 0,55-0,60 m unter Oberfläche wahrscheinlich ebenfalls auf Einfrachtung zurückzuführen. Zum Hangenden wieder zunehmende Anteile von Ammonia batavus sprechen für diese Deutung.

Die bathymetrische Obergrenze der Eggerelloides scaber-Zone entspricht nach der heutigen Verbreitung in der Nordsee eher der Sturmwellenbasis (SWB: LIEBAU 1980) als der Obergrenze des Schelfschlickes (Reineck et al. 1968). Liebau (1980) gibt als Tiefe für die Sturmwellenbasis in Nebenmeeren "meist zwischen 20 und $40 \mathrm{~m}$ “ an, was gut mit den Beobachtungen in der Deutschen Bucht übereinstimmt. Palökologisch spiegelt sich die Sturmwellenbasis nach Liebau u. a. in der oberen Verbreitungsgrenze kymatophober, d. h. die Wellenbewegung meidender Mikrobenthonten, wozu auch Eggerelloides scaber gehört. Nach Laboruntersuchungen von RichteR 1967: 309) hält sich E. scaber in den obersten Sedimentschichten auf, wodurch sie empfindlich gegenüber der Wasserbewegung ist. Die oben geäußerte Vermutung, daß diese Art im oberen Teil der von Exon (1972) beschriebenen scaber-reichen Schichten (Teil der „Einheit G“ WINN 1974) in der Ostsee wie in der Bg. H $15-3 \mathrm{~V}$ in der Nordsee umgelagert sein dürfte, findet somit eine experimentelle Stütze.

Zusammenfassend ist festzustellen, daß mit quantitativen und semiquantitativen Analysen vorläufig folgende 8 ökostratigraphische Foraminiferen-Zonen im Holozän der Deutschen Bucht unterschieden und bestimmten Milieus zugeordnet werden können:

1. Ammonia batavus var. a-Ókozone: Lagune (miohalin bis ?mesohalin); Teilsequenz 1. Monospezifische bis sehr artenarme Foraminiferen-Fauna. In der Deutschen Bucht erstmals in Bg. 1701 nachgewiesen (1,45-1,50 m unter Oberfläche $\hat{=} 30,45-30,50 \mathrm{~m}$ unter SKN).

Nach KNUDSEN (1979) ist diese von Woszidlo (1962) beschriebene Varietät möglicherweise synonym mit Ammonia aberdoveyensis HAYnes 1973.

2. Elphidium gunteri-Ơkozone: Lagune bis brandungsgeschütztes Schlickwatt (mio- bis brachyhalin/euhalin). Unterteilung in 3 Ökosubzonen ist möglich (s. Abb. 3); Teilsequenzen qhK $1-2$.

Artenarme Foraminiferen-Fauna mit $>5 \%$ Elphidium gunteri.

Miliammina fusca, die nach Richter (1967: 301) ebenfalls typisch für Watten „mit im allgemeinen geringer Turbulenz und schwachen Strömungen“ ist, hat offenbar in den entsprechenden Paläo-Milieus der Deutschen Bucht keine Bedeutung als Leitform.

Brackwasser-anzeigende Sandschaler wie Trochammina inflata und Trochammina macrescens ( $\hat{=}$ "Jadammina polystoma") zeigen gelegentlich Einflüsse von bzw. Übergänge zu Marsch-Milieus an.

3. Elphidium williamsoni-Ökozone: Lagune (einschließlich Ufer), brandungsgeschütztes Sandwatt (pliohalin bis euhalin); Teilsequenzen qhK 1-2. Nach Richter (1967: 301,

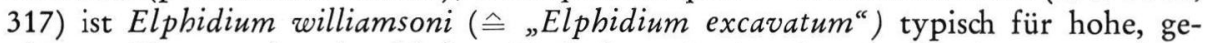
schützte Watten ohne brackischen Einfluß; meist ist sie mit Nonion germanicum (스 „Nonion depressulum“) vergesellschaftet. Da der Anteil an Elphidium williamsoni bereits in rezenten Taphozönosen unter $20 \%$ absinken kann, werden die entsprechenden fossilen Milieus der Deutschen Bucht mit Mindestprozentanteilen von $>10 \%$ definiert.

Nach Lutze (1968), Scott \& Medioli (1980) u. a. ist E. williamsoni (今 „Cribrononion articulatum $\hat{=}$ "Cribrononion umbilicatulum") mit weniger als $10 \%$ an der Foraminiferen-Fauna im tiefsten Teil der lagunären Vegetationszone bzw. der Marsch vertreten. 
In Bg. 1701 kommt die williamsoni-Zone im Hangenden der Ammonia batavus var.aZone in 0,65-1,30 m unter Oberfläche in den Teilsequenzen qhK1 und qhK2 vor. In Bg. 1705 wurde sie in 1,30-2,05 m unter Oberfläche ( $\triangleq 38,30-39,05 \mathrm{~m}-\mathrm{SKN})$ festgestellt.

4. Elphidium excavatum (forma selseyense)-OKozone: offenes Watt (euhalin bis brachyhalin); Teilsequenz qhK2.

Diese OKkozone ist charakterisiert durch Elphidium excavatum mit $>10 \%$-Anteilen an einer individuenreichen Foraminiferen-Fauna zusammen mit hohen Prozentanteilen an Nonion germanicum (meist weit über $20 \%$ ). Schwach skulptierte, kleine bis mittelgroße Morphotypen von Ammonia batavus herrschen vor. Elphidium gunteri tritt selten auf und erreicht selten mehr als $5 \%$ der Fauna.

Diese Kriterien entsprechen den aktuopaläontologischen Befunden RichTER's (1967: 303) sowohl auf den „offenen, tiefen Watten brandungsgeschützter Küsten“ als auch auf den „brandungsexponierten Watten zwischen HWL und NWL“.

In Bg. 297 gehört der höchste Teil der Watt-Sedimente (Teilsequenz qhK2) in die excavatum-Zone (s. Abb. 5).

Die individuenarme Foraminiferen-Assoziation im Vorstrand-Bereich der offenen Ostsee, die nach LUTZE (1968) > 10\% an excavatum enthält, dürfte fossil bereits der „allochthonen küstennahen Biofazies" entsprechen.

5. „Allochthone küstennahe Biofazies“: Vorstrand, Übergangszone zwischen Küstensand und Schelf (oberer Teil); Teilsequenzen qhK2-3.

Individuen- und artenarme Foraminiferen-Faunen aus großwüchsigen, robusten, meist schlecht erhaltenen Formen charakterisieren diesen Bereich. Qualitativ unterscheidet sich diese Ökozone nicht von der Ökozone der "Abroll-Foraminiferen“ (s. u.).

Von einer „allochthonen Vorstrand-Biofazies“ im Sinne von HERTweck (1971b) wird hier deshalb nicht gesprochen, weil diese individuen- und artenarmen ForaminiferenFaunen nicht auf den Vorstrand-Bereich beschränkt sind.

Die Mehrzahl der in diesem Milieu lebenden Foraminiferen wird in die benachbarten Rinnen, die Übergangszone zum Schelf oder die brandungsexponierten Watten umgelagert.

Die „allochthone küstennahe Biofazies" kennzeichnet Teile der qhK2-Teilsequenz (z. B. Bg. 1702: Probe 1,25-1,30 m unter Oberfläche $\triangleq 34,25-34,30 \mathrm{~m}-\mathrm{SKN}$ ).

6. O’kozone der „Abroll-Foraminiferen“ (Ammonia batavus-Ökozone):

Rinnen, Ubbergangszone zwischen Küstensand und Schelf; Teilsequenzen qhK2-3. Diese Ókozone ist gekennzeichnet durch zahlreiche große, reich skulptierte AmmoniaGehäuse, die Liebau (1978) als „Abroll-Foraminiferen“ bezeichnete. In diese Zone gehören individuenreiche, aber artenarme Foraminiferen-Faunen, in denen kleine und zartschalige Watt- und Nordsee-Arten \pm fehlen. Großwüchsige Formen von Ammonia batavus, Quinqueloculina seminulum, Elphidium williamsoni, E. excavatum und Nonion germanicum beherrschen in wechselndem Mengenverhältnis das Bild dieser Ơkozone.

Richter (1967: 305) beschrieb entsprechende Taphozönosen aus Rinnen-Sedimenten, wobei er auf die weitgehende Allochthonie dieser Zonen hinwies.

Wie oben dargelegt (s. Bg. 297, Abb. 5), kann diese Ókozone auch im unteren Teil der qhK3-Teilsequenz auftreten. Offenbar kennzeichnet diese Ökozone dort umgelagerte Küstensande, die aus dem Vorstrand-Bereich unter die Sturmwellen-Basis verfrachtet wurden. Im Hangenden folgt nach Foraminiferen die Übergangszone. 
7. Übergangszone: Übergangszone zwischen Küstensand und Schelf (sensu REINECK \& SiNGH 1973/1980: "offshore-beach transition", s. Abb. 4), küstenferner Bereich in der Nähe der Sturmwellen-Basis; qhK3-Teilsequenz, selten Teilsequenzen qhK1-2.

Individuen- und artenreiche Foraminiferen-Fauna mit Eggerelloides scaber $>5 \%$ und zahlreichen, meist kleinen Arten des offenen Meeres (Asterigerinata mamilla, Bolivina pseudoplicata, Bulimina gibbalelongata, Brizalina pseudopunctata, Fursenkoina fusiformis u. a.), die ebenfalls in geringer Individuenzahl vorkommen. Ammonia batavus und Elphidium excavatum sind die häufigsten Arten mit zusammen meist $>50 \%$ der Fauna. Nach aktuopaläontologischen Untersuchungen von RicHTER (1967: 308) liegt die „Übergangszone" im Gebiet von Außenjade und Außenweser in Wassertiefen von $15-20 \mathrm{~m}$. Die Ubergangszone ist in der qhK3-Teilsequenz stets anzutreffen.

8. Eggerelloides scaber-Ökozone: Schelfbereiche unterhalb der Sturmwellen-Basis, Ruhigwasser-Milieus (Schelfbecken); qhK3-Teilsequenz.

Okozone mit $>5 \%$ Eggerelloides scaber in der Foraminiferen-Fauna. Fauna meist artenärmer als in Übergangszone, aber mit etwas höherem Anteil an kleinen Kalkund Sandschalern.

Nach Richter (1967: 309) ist Eggerelloides (스 „Eggerella“) scaber rezent besonders häufig in nährstoffreichen, schlickigen Sedimenten. Die darüber hinausreichende Verbreitung bis in sehr gut sortierte Feinsande (s. JARKE 1961: 27) scheint zumindest teilweise durch Erosion und Umlagerung verursacht zu sein. Lutze (1965: 93) nennt Salzgehalte von mindestens $20 \%$,wenigstens für einen Teil des Jahres “ für diese Art. Dementsprechend bleibt die Art in der Ostsee vorwiegend auf den Einstrombereich südlich des Großen Belts und das angeschlossene Rinnensystem beschränkt.

Die scaber-Zone kommt in weiter Verbreitung sowohl in der qhK3-Teilsequenz im Gebiet des Elbe-Urstromtales als auch in den benachbarten Arealen mit hochliegendem Pleistozän vor.

Die Ơkozonen entsprechen der Rangordnung nach, meist aber nicht ihrer Reichweite nach den Fazieseinheiten (i. S. von Barckhausen et al. 1977). Die Fazieseinheiten werden lithologisch bzw. lithofaziell definiert, die Ökozonen palökologisch. Die Fazieseinheiten gehören somit dem lithostratigraphischen, die OKozonen dem biostratigraphischen Ordnungsprinzip i.w.S. an.

\section{Lithostratigraphische und fazielle Ergebnisse}

Während sich die bisherige stratigraphische Gliederung der oberflächennahen quartären Schichtenfolge im deutschen Nordsee-Sektor weitgehend an diejenige von Oele (1969) anlehnte (vgl. Sindowski 1970; Behre, Menke \& Streif 1979; Streif \& Uffenorde 1980), wurde in Kap. 2 eine neue auf litho- und biofaziellen Merkmalen basierende stratigraphische Gliederung entworfen, die sich an die von Barckhausen, Preuss \& Streif (1977) gegebene Gliederung des Küstenholozäns seewärts anschließt, und die bislang vorliegenden biostratigraphischen Daten genannt.

Ziel dieser neuen Gliederung ist es: (1) Verschwommen definierte feldgeologische Einheiten wie "Young Seasand" und (2) zeitabhängige, lithostratigraphische Einheiten wie "Elbow deposits" durch lithostratigraphische bzw. fazielle Einheiten zu ersetzen, zu untergliedern und zu präzisieren, um den Ablauf des holozänen Transgressionsgeschehens regional und lokal in den einzelnen Bohrprofilen besser rekonstruieren zu können, detailliertere Angaben über die Verbreitung der einzelnen Fazieskörper, über deren Genese, über Umlagerungsprozesse oder über die hydrodynamische Entwicklung der Deutschen Bucht zu gewinnen. 
Die hier vorgeschlagene Gliederung zeigt folgende Besonderheiten — insbesondere gegenüber der niederländischen (s. Abb. 1):

1. Die Gliederung basiert - entsprechend der WALTER'schen Regel - auf einer paläogeographischen, hydrodynamischen Gliederung eines Flachmeeres seewärts der Küstenmoore. Dabei lassen sich in Anlehnung an IRwIN (1965) drei Großräume mit unterschiedlichen hydrodynamischen Bedingungen auseinanderhalten: (1) der küstennahe Bereich mit fehlender bis geringer Wasserzirkulation und Gezeitenbewegung (qhK1: z. B. lagunärer Bereich), (2) der küstennahe Bereich, der überwiegend von den Kräften der Gezeiten- und Wellenbewegung beeinflußt wird (qhK2: Watt-Bereich, Bereiche der R.innen und Platen, Vorstrand-Bereich usw.) und (3) der küstenferne Bereich seewärts des durch Wellen und Gezeiten geprägten Raumes, der vorwiegend von Strömungen und Suspensionsvorgängen beeinflußt wird (qhK3: z. B. Übergangsbereich Küstensand/Schelf, Schelf-Bereich). Der Bereich 2 enthält außer den Wattablagerungen das gesamte Spektrum von Sanden, die von Reineck \& SingH (1973/1980) als Küstensande bezeichnet wurden. Grobklastische Residualsedimente weisen auf Aufarbeitung hin. Das niederländische Gliederungsschema entspricht zwar im Prinzip diesem paläogeographischen, hydrodynamischen Modell, die brackischen bis marinen Ablagerungen des Bereiches 1 scheinen jedoch entweder zu fehlen oder in den "Elbow/Calais-deposits" (Bereich 2) mit einbezogen worden zu sein (s. Jelgersma, Oele \& Wiggers 1979).

2. Eine Abtrennung der klastischen Süßwasser-Ablagerungen von brackischen und brakkisch-marinen Ablagerungen lagunärer Milieus ist lithostratigraphisch kaum durchführbar, sondern verlangt eine biofazielle oder geochemische Detailuntersuchung. Gleiches gilt für Unterscheidung brackischer und mariner Sedimente. In der Praxis wird jedoch oft eine lithostratigraphische Gliederung ohne komplizierte und zeitaufwendige Spezialanalysen gewünscht. Die vorliegende Gliederung trägt diesem Bedürfnis Rechnung, indem salinitätsunabhängige Kriterien die lithostratigraphische Zuordnung bestimmen.

3. Der Süßwasserton, der von Oele (1969) zur "Lower Peat Formation" gerechnet wurde, wird der Klastischen Sequenz (qhK) zugeordnet.

4. Die küstennah-marinen Schluffe, Feinsande und Mittelsande der qhK2-Teilsequenz stimmen meist recht gut mit der Beschreibung der "Elbow deposits" von Oele überein. Übereinstimmung besteht auch - mit einigen Ausnahmen — in der Entwicklung von feinkörnigen (tonig-schluffigen) zu mittelkörnigen (fein- bis mittelsandigen) Sedimenten zum Hangenden hin. Vollmarine Paläo-Milieus, größtenteils vollmarine WattAblagerungen scheinen in beiden Gebieten in diesen Schichten vorzuherrschen. Dem lithostratigraphischen Charakter dieser Schichtfolge entsprechend, läßt sich keine Trennung in präboreale bis boreale "Elbow deposits" und atlantische bis früh-subboreale "Calais-deposits" durchführen, weshalb z. B. Jelgersma, Oele \& Wiggers (1979) folgerichtig von "Elbow/Calais deposits" sprechen. Zu den küstennahen BewegtwasserAblagerungen gehören möglicherweise auch die von Wunderlich (1980: $216 \mathrm{f}$., Abb. 3) abgebildeten Schichten im Liegenden einer „scharf ausgebildeten Erosionsdiskordanz“. Palynologische und mikropaläontologische Untersuchungen stehen noch aus.

5. Die Schichten über dieser Erosionsdiskordanz entsprechen den küstenfernen Schluffen, Fein- und Mittelsanden der qhK3-Teilsequenz, deren fazielle Äquivalente von Oele (1969) als Junger Seesand bezeichnet wurden. Dieses Schichtglied lagert in der südlichen Nordsee verbreitet auf verschieden alten Schichten.

Molluskenschille, Konglomerate oder auch nur erhöhter Schalengehalt und gröbere Körnung markieren seine Basis und bilden damit den besten lithostratigraphischen Leithorizont im Holozän der südlichen Nordsee. Zusammensetzung, Aufbau und Ânn- 
lichkeit mit rezenten Sturmablagerungen in der Deutschen Bucht (s. ReINeck et al. 1968, Gadow \& Reineck 1969, Reineck \& Singh 1972) lassen auf Tempestit-Genese schließen. Sturm-Ereignisse bestimmen auch verbreitet den oberen Teil der qhK3-Teilsequenz, so daß eine fazielle Unterteilung der Teilsequenz nach Vorkommen oder Fehlen von Sturmablagerungen oftmals möglich ist. Die küstenfern-marine Teilsequenz erreicht ihre größten Mächtigkeiten offenbar in mehreren getrennten Abschnitten des „Elbe-Urstromtales“: so liegt ein Maximum S Helgoland, ein weiteres auf und SE der Weißen Bank (vgl. Figge 1980).

6. Am Beispiel des Kerns 26 der Station 3 am Südlichen Schillgrund (niederländischer Nordseesektor, s. Kolp 1976), der paläontologisch sehr detailliert bearbeitet wurde (s. Behre \& Menke 1969, Pazotka von Lipinski \& Wiegank 1969, Diebel \& PietrZENIUK 1971) und hier deshalb eingehend diskutiert wurde, wird deutlich, daß sich die ökostratigraphischen Grenzen nicht mit denen der vorgeschlagenen lithostratigraphischen Gliederungen zu decken brauchen. Palökologische Untersuchungen, insbesondere der Foraminiferen-Faunen, erweisen sich als ein wichtiges Hilfsmittel zur Identifizierung der Paläomilieus. Unterschiede in der Verbreitung euhaliner Foraminiferen in den Teilsequenzen qhK1 und 2 werden auf unterschiedlich weites Vordringen von Wassermassen des offshore-Bereiches in die küstennahen Milieus gedeutet. Ostracoden und Mollusken sind nur in einzelnen Abschnitten der Klastischen Sequenz häufig genug, um statistisch gesicherte quantitative palökologische Befunde zu liefern. Besonderes Augenmerk ist auf umgelagertes paläontologisches, darunter insbesondere auch palynologisches Material zu richten, das meist eine küstennähere Entstehung vortäuscht, ja bei Pollenuntersuchungen sogar ein höheres Alter der Schichten ergeben kann.

7. Weitgehend in Übereinstimmung mit aktuopaläontologischen Befunden können die Holozän-Profile mit Foraminiferen gegliedert und interpretiert werden. Durch quantitative, teilweise bereits durch semiquantitative Analysen lassen sich vorläufig 8 ökostratigraphische Zonen unterscheiden und bestimmten Milieus zuordnen. Dadurch kann der Transgressionsverlauf in der Klastischen Sequenz präzisiert und die räumliche Entwicklung der einzelnen Ablagerungsmilieus während des Meeresspiegelanstieges rekonstruiert werden.

\section{Dank}

Die Mehrzahl der oben erwähnten Bohrungen stammt aus dem Lagerstätten-Projekt der Arbeitsgemeinschaft (ARGE) „Steine und Erden im Nordsee-Schelfbereich“, in dem 1974 und 1975 unter Federführung des NLfB (Hannover) und unter Leitung von Dr. G. LUDWIG insgesamt 291 Flachbohrungen mit einem englischen Vibrationskernbohrgerät niedergebracht wurden. Die von Ludwig, Müller \& STREIF $(1979,1982)$ begonnene wissenschaftliche Auswertung dieses umfangreichen Rohmaterials wird hiermit fortgesetzt. Den an der ehemaligen ARGE beteiligten Firmen HOCHTIEF AG (Essen), HULSKENS \& CO (Wesel), HÜRTHERBERG STEINE UND ERDEN GMBH (Köln) NORDSEEKIES GMBH (Hannover), WESTMINSTER GRAVELS GMBH (Bremen) und der Firmengruppe ROHRS \& CO (Bremen), IBAG-VERTRIEB GMBH (Neustadt/Weinstraße), WESLING KG (Münchehagen) und EGGERSMANN KG (Rinteln) sowie der PREUSSAG AG (Hannover) gilt mein besonderer Dank für die Erlaubnis zur Veröffentlichung dieser Untersuchung. Die Herren Dres. G. Ludwig, H. Streif und R. Vinken, Herr K.-J. Meyer, Herr W. Possin (NLfB, Hannover) und Herr Dr. H. Mülier (BGR, Hannover) haben wesentlichen Anteil an dem Zustandekommen dieser Publikation. Ihnen 
wie auch Herrn Dr. K. FIGGE und dem Deutschen Hydrographischen Institut (Hamburg) sei herzlich gedankt. Besonderer Dank gilt Herrn Dr. Streif für die kritische Durchsicht des Manuskriptes.

Die Deutsche Forschungsgemeinschaft ermöglichte dankenswerterweise die vorliegende Untersuchung durch finanzielle Förderung in dem Forschungsvorhaben Vi 32/23 und 27.

\section{Schriftenverzeichnis}

Averdieck, F.-R. (1972): Palynologische Untersuchungen an Bohrungen in der Flensburger Außenförde (Ostsee). - Meyniana, 22: 1-4; Kiel.

Barckhausen, J., Look, E.-R., Vinken, R. \& Voss, H.-H. (1975): Symbolschlüssel Geologie. Symbole für die Dokumentation und Automatische Datenverarbeitung - ADV - geologischer Feld- und Aufschlußdaten. - Niedersächs. L.-Amt Bodenforsch. u. B.-Anst. Geowiss. Rohstoffe (Hrsg.): 1-135; Hannover (2. Aufl.).

- Preuss, H. \& Streif, H. (1977): Ein lithologisches Ordnungsprinzip für das Küstenholozän und seine Darstellung in Form von Profiltypen. - Geol. Jb., A 44: 45-77; Hannover.

Behre, K.-E. \& Menke, B. (1969): Pollenanalytische Untersuchungen an einem Bohrkern der südlichen Doggerbank. - Beitr. Meereskde., 24/25: 122-129; Berlin.

-, - \& StreIF, H. (1979): The Quaternary geological development of the German part of the North Sea. - In: Oele, E., Schüttenhelm, R. T. E. \& Wiggers, A. J. (eds.): The Quaternary history of the North Sea. Acta Univ. Ups. Symp. Univ. Ups. Annum Quingentesimum Celebrantis, 2: 85-113; Uppsala.

Culver, S. J. \& Banner, F. T. (1978): Foraminiferal assemblages as Flandrian palaeoenvironmental indicators. - Palaeogeogr. Palaeoclimatol. Palaeoecol., 24 (1): 53-72, 8 figs.; Amsterdam.

Diebel, K. \& Pietrzeniuk, E. (1971): Holozäne Ostracoden von der Doggerbank, Nordsee. In: Oertli, H. J. (ed.): Paléoécologie Ostracodes, Pau, 1970, Bull. Centre Rech. Pau-SNPA, 5 suppl.: 377-390; Pau.

DörJes, J. (1971): Der Golf von Gaeta (Tyrrhenisches Meer). IV. Das Makrobenthos und seine küstenparallele Zonierung. - Senckenbergiana maritima, 3: 203-246; Frankfurt a. M.

- \& Hertweck, G. (1975): Recent biocoenoses and ichnocoenoses in shallow-water marine environments. - In: Frey, R. W. (ed.): The study of trace fossils: 459-491; New York/ Heidelberg/Berlin (Springer).

- Gadow, S., Reineck, H.-E. \& Singh, I. B. (1970): Sedimentologie und Makrobenthos der Nordergründe und der Außenjade (Nordsee). - Senckenbergiana marit., 2: 31-59; Frankfurt a. M.

Exon, N. (1972): Sedimentation in the outer Flensburg Fjord area (Baltic Sea) since the last glaciation. - Meyniana, 22: 5-62; Kiel.

FigGe, K. (1980): Das Elbe-Urstromtal im Bereich der Deutschen Bucht. — Eiszeitalter u. Gegenwart, 30: 203-211; Hannover.

GaDow, S. \& Reineck, H.-E. (1969): Ablandiger Sandtransport bei Sturmfluten. - Senckenbergiana marit., 50: 63-78; Frankfurt a. M.

HAAKe, F. W. (1962): Untersuchungen an der Foraminiferen-Fauna im Wattgebiet zwischen Langeoog und dem Festland. - Meyniana, 12: 25-64, 9 Abb., 5 Tab., 6 Taf.; Kiel.

Hanisch, J. (1980): Neue Meeresspiegeldaten aus dem Raum Wangerooge. - Eiszeitalter u. Gegenwart, 30: 221-228; Hannover.

HAYNES, J. R. (1973): Cardigan Bay Recent Foraminifera (Cruises of the R.V. Antur, 1962-1964). - Brit. Mus. (Nat. Hist.) Bull., Zool., Suppl., 4: 1-245, pls. 1-33, text figs. 1-47; London.

Hedberg, H. D. (Ed.) (1976): International stratigraphic guide. - 1-200; New York/London/ Sydney/Toronto (J. Wiley \& Sons). 
Hertweck, G. (1971 a): The animal community of a muddy environment and the development of biofacies as effected by the life cycle of characteristic species. - In: Crimes, T. P. \& Harper, J. C. (eds.): Trace fossils: 235-242; Liverpool (Geol. J., spec. Iss.).

- (1971 b): Der Golf von Gaeta (Tyrrhenisches Meer). V. Abfolge der Biofaziesbereiche in den Vorstrand- und Schelfsedimenten. - Senckenbergiana maritima, 3: 247-276; Frankfurt a. M.

- \& Reineck, H.-E. (1969): Sedimentologie der Meeresbodensenke NW von Helgoland (Nordsee). - Senckenbergiana maritima, (1) 50: 153-164; Frankfurt a. M.

Hiltermann, H. (1966): Klassifikation rezenter Brack- und Salinar-Wässer in ihrer Anwendung für fossile Bildungen. - Z . dt. geol. Ges., 115: 463-469; Hannover.

IRwin, M. L. (1965): General theory of epeiric clear water sedimentation. - Bull. Amer. Assoc. Petrol. Geol., 49 (4): 445-459; Tulsa, Okla.

Jansen, J. H. F., Van WeEring, Tj. C. E. \& Eisma, D. (1979): Late Quaternary sedimentation in the North Sea. - In: Oele, E., Schüttenhelm, R. T. E. \& Wiggers, A. J. (eds.): The Quaternary history of the North Sea. - Acta Univ. Ups. Symp. Univ. Ups. Annum Quingentesimum Celebrantis, 2: 175-187; Uppsala.

JARKE, .T. (1961): Die Beziehungen zwischen hydrographischen Verhältnissen, Faziesentwicklung und Foraminiferenverbreitung in der heutigen Nordsee als Vorbild für die Verhältnisse während der Miocän-Zeit. - Meyniana, 10: 21 - 36, 9 Abb., 4 Taf.; Kiel.

Jelg :rsma, S., Oele, E. \& W. Iggers, A. J. (1979): Depositional history and coastal development in the Netherlands and the adjacent North Sea since the Eemian. In: Oele, E., SchütTenHelm, R. T. E. \& Wiggers, A. J. (eds.): The Quaternary history of the North Sea. - Acta Univ. Ups. Symp. Univ. Ups. Annum Quingentesimum Celebrantis, 2: 115-142; Uppsala.

Kolp, O. (1974): Submarine Uferterrassen in der südlichen Ost- und Nordsee als Marken eines stufenweise erfolgten holozänen Meeresspiegelanstiegs. - Baltica, 5: 11-40; Vilnius.

- (1975): Die submarinen Uferterrassen der südlichen Ost- und Nordsee und ihre Beziehungen zum eustatischen Meeresspiegelanstieg. - Beitr. Meereskde., 35: 1-48; Berlin.

- (1976): Submarine Uferterrassen der südlichen Ost- und Nordsee als Marken des holozänen Meeresspiegelanstiegs und der Úberflutungsphasen der Ostsee. - Petermanns Geogr. Mitt., 120 (1): 1-23; Gotha/Leipzig.

Laban, (1978): Holocene sediments on the Dutch part of the continental shelf. - Vortrag, NW Europ. Shelf Quat. Symp. \& Workshop, Edinburgh 9.-10. 3. 1978 (Mskr., unveröff.).

Liebau, A. (1978): Abrieb-geschützte Foraminiferen. - N. Jb. Geol. Paläont. Abh., 157: 119-122; Stuttgart.

- (1980): Paläobathymetrie und Ókofaktoren: Flachmeer-Zonierungen. - N. Jb. Geol. Paläont. Abh., 160 (2): 173-216; Stuttgart.

Ludwig, G. (1975): Schichtenverzeichnis der Bohrungen Nr. 30 bis 355 im Nordsee-Schelfbereich. MS „Irene S“-Fahrten 1974. - Arch. Ber. NLfB: 89 S., Hannover (unveröff.).

- Müllek, H. \& Streif, H. (1979): Neuere Daten zum holozänen Meeresspiegelanstieg im Bereich der Deutschen Bucht. - Geol. Jb., D 32: 3-22, 2 Abb., 7 Tab.; Hannover.

- _ \& - (1981): New dates on Holocene sea-level changes in the German Bight. - Spec. Publ. int. Ass. Sediment., 5: 211-219; Liverpool.

Lutze, G. F. (1965): Zur Foraminiferen-Fauna der Ostsee. - Meyniana, 15: 75-147, 32 Abb., 15 Taf.; Kiel.

- (1968): Jahresgang der Foraminiferen in der Bootsand-Lagune. - Meyniana, 18: 13-30; Kiel.

- (1974): Foraminiferen der Kieler Bucht (westliche Ostsee): 1. „Hausgartengebiet“ des Sonderforschungsbereich 95 der Universität Kiel. - Meyniana, 26: 9-22; Kiel.

Meyer, K.-J. (1980): Bericht über pollenanalytische Untersuchung: H 15/2 V. - Arch.-Ber. NLfB: 2 S.; Hannover (unveröff.).

- (1981 a): Bericht über pollenanalytische Untersuchungen: Bhrg. N 168, N 188, N 210, N 245, Arch.-Ber. NLfB: 3 S.; Hannover (unveröff.).

- (1981 b): Bericht über pollenanalytische Untersuchungen: Bhrg. 270, Bhrg. 340. - Arch.-Ber. NLfB: 2 S.; Hannover (unveröff.).

MülLER, H. (1981 a): Palynologische Untersuchung von 2 Kernproben der Nordsee-Bohrung 133. - Arch.-Ber. BGR: 2 S.; Hannover (unveröff.).

- (1981 b): Palynologische Untersuchung von 3 Kernproben der Nordsee-Bohrung 85 . Arch.-Ber. BGR: 2 S.; Hannover (unveröff.).

Murray, J. W. (1973): Distribution and ecology of living benthic foraminiferids. - 1-274; London (Heinemann Educational Books). 
Oele, E. (1969): The Quaternary Geology of the Dutch part of the North Sea, north of the Frisian isles. - Geol. Mijnbouw, 48 (5): 467-479; s'Gravenhage.

- Late Quaternary geology of the North Sea south-east of the Dogger Bank. - In: Delany, F. M. (ed.): The Geology of the East Atlantic Continental Margin, 3, Inst. Geol. Sci., 70/15: 25-34; London.

- (1971 b): The Quaternary geology of the southern area of the Dutch part of the North Sea. - Geol. Mijnbouw, 50 (3): 461-474; Delft.

Overbeck, F. (1975): Botanisch-geologische Moorkunde unter besonderer Berücksichtigung der Moore Nordwestdeutschlands als Quellen der Vegetations-, Klima- und Siedlungsgeschichte. 1-719; Neumünster (Wachholtz).

Pazotka von Lipinski, G. \& Wiegank, F. (1969): Foraminiferen aus dem Holozän der Doggerbank. - Beitr. Meereskde., 24-25 (III): 130-174, 3 Abb., 14 Taf.; Berlin.

Reineck, H.-E., DörJes, J., Gadow, S. \& Hertweck, G. (1968): Sedimentologie, Faunenzonierung und Faziesabfolge vor der Ostküste der inneren Deutschen Bucht. - Senckenbergiana lethaea, 49: 261-309; Frankfurt a. M.

- \& SingH, I. B. (1972): Genesis of laminated sand and graded rhythmites in storm-sand layers of shelf-mud. - Sedimentol., 18: 123-128; Amsterdam.

- \& - (1973): Depositional sedimentary environments. - 1-439; Berlin/Heidelberg/New York (Springer-Verl.).

... \& - (1980): Depositional sedimentary environments. - 2. Aufl., 1-549; Berlin/Heidelberg/New York (Springer-Verl:).

Richter, G. (1961): Beobachtungen zur Ókologie einiger Foraminiferen des Jade-Gebietes. Natur u. Volk, 91 (5): 163-170, 7 Abb.; Frankfurt a. M.

- (1964 a): Zur Ókologie der Foraminiferen. I. Die Foraminiferen-Gesellschaften des Jadegebietes. - Natur u. Mus., 94 (9): 343-353, 14 Abb.; Frankfurt a. M.

- (1964 b): Zur Ókologie der Foraminiferen. II. Lebensraum und Lebensweise von Nonion depressulum, Elpbidium excavatum und Elphidium selseyense. - Natur u. Mus., 94 (11): 421-430, 4 Abb., 3 Tab.; Frankfurt a. M.

- (1965): Zur Ớkologie der Foraminiferen. III. Verdriftung und Transport in der Gezeitenzone. - Natur u. Mus., 95 (2): 51-62, 4 Abb., 3 Tab..: Frankfurt a. M.

- (1967): Faziesbereiche rezenter und subrezenter Wattensedimente nach ihren ForaminiferenGemeinschaften. - Senck. leth., 48 (3/4): 291-335; Frankfurt a. M.

SCHÄFER, W. (1963): Biozönose und Biofazies im marinen Bereich. - Aufsätze und Reden Senck. Naturforsch. Ges., 11: 1-37; Frankfurt a. M.

Scott, D. B. \& Medioli, F. S. (1978): Vertical zonations of marsh foraminifera as accurate indicators of former sea levels. - Nature, 272 (5653): 528 - 531; London.

- \& - (1980): Quantitative studies of marsh foraminiferal distributions in Nova Scotia: implications for sea level studies. - Cushman Found. Foram. Res., spec. publ., 17: 1-58, 22 figs., 5 pls., $4+14$ tabs.; Menlo Park, Cal.

Sindowskr, K.-H. (1970): Das Quartär im Untergrund der Deutschen Bucht (Nordsee). - Eiszeitalter u. Gegenwart, 21: 33-46; OOhringen/Württ.

Streif, H. (1978): A new method for the representation of sedimentary sequences in coastal regions. - Proc. 16th Coastal Engineering Conf. ASCE/Hamburg 1978: 1245-1256; Hamburg.

- (1979): Die Profiltypenkarte des Holozän - eine neue geologische Karte zur Darstellung von Schichtenfolgen im Küstenraum für praktische und wissenschaftliche Zwecke. - Die Küste, 34: 79-86; Heide i. H.

- \& UfFENORDE, H. (1980): 1. Zwischenbericht zum Forschungsvorhaben „Untersuchungen zum pleistozänen und holozänen Transgressionsgeschehen im Bereich der südlichen Nordsee“. DFG-Ber.: 1-32; Hannover (unveröff.).

- \&-(1981):2. Zwischenbericht zum Forschungsvorhaben „Untersuchungen zum pleistozänen und holozänen Transgressionsgeschehen im Bereich der südlichen Nordsee“. - DFG-Ber.: 1-29; Hannover (unveröff.).

Wefer, G., Weber, M. \& ERLEnKeuser, H. (1978): Sandablagerungen während der postglazialen Transgression in der Eckernförder Bucht (westliche Ostsee). - Senckenbergiana marit., 10 (1/3): 39-61; Frankfurt a. M.

WINN, K. (1974): Present and Postglacial Sedimentation in the Great Belt Channel (Western Baltic). - Meyniana, 26:63-101; Kiel.

Wunderlich, F. (1980): Transgression und Umlagerung im Gebiet des Helgoland-Riffs. - Eiszeitalter u. Gegenwart, 30: 213-219; Hannover. 